\title{
Article
}

\section{Thin film structural analysis using variable-period $x$-ray standing waves}

Gibson, Joshua S., Syres, Karen, Buckley, Matthew, Lee, Tien-Lin, Thakur, Pardeep K. and Jones, Robert G.

Available at https://clok.uclan.ac.uk/24365/

Gibson, Joshua S., Syres, Karen orcid iconORCID: 0000-0001-7439-475X, Buckley, Matthew, Lee, Tien-Lin, Thakur, Pardeep K. and Jones, Robert G. (2018) Thin film structural analysis using variable-period $x$-ray standing waves. Physical Review B, 98 (16). ISSN 2469-9950

It is advisable to refer to the publisher's version if you intend to cite from the work. http://dx.doi.org/10.1103/PhysRevB.98.165402

For more information about UCLan's research in this area go to http://www.uclan.ac.uk/researchgroups/ and search for <name of research Group>.

For information about Research generally at UCLan please go to http://www.uclan.ac.uk/research/

All outputs in CLoK are protected by Intellectual Property Rights law, including Copyright law. Copyright, IPR and Moral Rights for the works on this site are retained by the individual authors and/or other copyright owners. Terms and conditions for use of this material are defined in the policies page.

\section{CLoK}

Central Lancashire online Knowledge www.clok.uclan.ac.uk

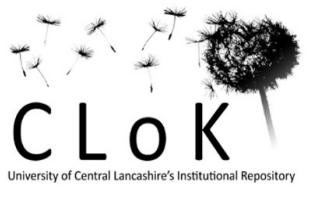




\section{Thin film structural analysis using variable period $X$-ray standing waves.}

Joshua S Gibson ${ }^{1}$, Karen L Syres ${ }^{1,3}$, Matthew Buckley ${ }^{1}$, Tien-Lin Lee ${ }^{2}$, Pardeep K Thakur $^{2}$ and Robert G Jones ${ }^{1 *}$

1 School of Chemistry, University of Nottingham, Nottingham NG7 2RD, UK

2 Diamond House, Harwell Science and Innovation Campus, Didcot, Oxfordshire OX11 0DE, UK

3 Current address: Jeremiah Horrocks Institute, University of Central Lancashire, Fylde Road, Preston, PR1 2HE

* Author to whom correspondence should be addressed.

robert.g.jones@nottingham.ac.uk

\section{Abstract}

Variable period X-ray standing wave (VPXSW) studies have been carried out using 3 keV X-rays and photoelectron detection. Two model surfaces have been used, a native $\mathrm{SiO}_{2}$ layer (20 ̊ thick) on bulk silicon, and a purpose built multilayer surface comprising a chloroform/water marker layer (12 $\AA$ thick) on an ionic liquid spacer layer (211 $\AA$ thick) deposited on $\mathrm{SiO}_{2} / \mathrm{Si}$ substrate at $90 \mathrm{~K}$. By using photoelectron detection, both chemical and elemental sensitivity were achieved. The surfaces were modelled using dynamic X-ray scattering for X-ray intensity, and attenuation of photoelectrons transmitted through the layers, to produce simulations which accurately reproduced the experimental VPXSW measurements. VPXSW measurements made using the substrate, spacer layer and marker layer photoelectron signatures produced consistent structural values. This work demonstrates that VPXSW can be used to determine chemically specific layer thicknesses within thick $(\$ 300 \AA$ ) surface structures composed of the light elements B, C, N, O, F and Cl with an accuracy of 10 to $15 \AA$, perpendicular to the surface.

PACS number(s) 68.49.Uv, 61.10.Kw, 68.35.-p, 68.35.Ct 


\section{INTRODUCTION}

The techniques available for determining the composition of thin films 1-50 nm thick, as a function of depth, are currently rather limited. Ellipsometry ${ }^{1-3}$ provides thickness and dielectric information, while X-ray reflectivity ${ }^{4}$ provides thickness and the electron density profile perpendicular to the surface. Chemical species identification can be achieved by ion stripping methods coupled with analysis. For example, secondary ion mass spectrometry, SIMS $^{5}$, or photoelectron spectroscopy with ion etching, but these are inherently destructive and the depth resolution is degraded by roughening. Angle resolved ARXPS can map depth distributions of chemically identifiable species, but the resolution of $\approx 0.81 z^{6}$, where $z$ is the depth, is rather low. X-ray standing wave (XSW) fields have been used to determine surface atomic positions ${ }^{7}$, and fall into two categories. i) photoelectrons, Auger electrons and secondary electrons have been used as the detection method for XSWs formed by Bragg reflection from crystalline samples to measure positions with high precision $(0.02 \AA)$ over rather short ranges $(<1 \mathrm{~nm})^{7,8}$, or over somewhat longer ranges using synthetic multilayer crystals 9,10 . ii) X-ray fluorescence detected variable period $\mathrm{XSW}$, where the standing wave is formed by reflection from a flat surface, has been used to measures positions over much longer scales, $10-100 \mathrm{sm}^{7,11}$, but only with elemental sensitivity.

X-ray standing waves have been used over the past several decades for structural analysis of both bulk solids and surfaces ${ }^{7}$. Generally a standing wave is formed when two waves of the same wavelength and a given phase relationship cross. An Xray standing wave can be formed by X-ray diffraction ${ }^{7,12-16}$ where the incident and diffracted X-ray beams overlap. For Bragg diffraction, $\lambda=2 d_{h k l} \sin \theta$, where $\lambda$ is the $\mathrm{X}$ ray wavelength, $d_{h k l}$ the spacing of the $(h k l)$ set of planes and $\theta$ the angle of incidence and reflection. The standing wave intensity has a fixed periodicity of $d_{h k l}$ along the normal to the $(h k l)$ planes. The phase difference between the incident and diffracted beam at the Bragg condition is changed by either scanning $\theta$ over a small angular range for a fixed $\lambda$, (normally referred to as XSW), or by scanning $\lambda$ (i.e. the X-ray energy) slightly for a fixed $\theta$. For the latter, $\theta$ is often set to $90^{\circ}$ and is known as normal incidence X-ray standing wave (NIXSW) where the sine function is at a turning point making the technique relatively insensitive to crystal imperfections, and 
hence applicable to rather poor crystals such as metal crystals. To the low energy side of the Bragg condition, only the incident X-ray beam exists as a travelling wave passing through the sample. As the Bragg condition is approached, the diffracted beam comes into existence and forms the standing wave with the nodes located on the $(h k l)$ planes and the antinodes half way between. As the Bragg condition is traversed, the phase relationship changes shifting the position of the standing wave until the antinodes are on the $(h k l)$ planes and the nodes are half way between. On the high energy side of the Bragg condition, the diffracted beam disappears, leaving just the incident beam passing through the sample. The standing wave exists both inside and outside of the crystal and its intensity and position can be accurately calculated using dynamical X-ray theory. The standing wave is the "ruler" by which atomic positions are measured by monitoring the photoelectrons, Auger electrons, secondary electrons and fluroescence X-ray photons from atoms within the wave field. By monitoring the intensity of these emissions, the positions of the atoms within the X-ray wave field are determined, and hence the positions relative to the $(h k l)$ planes. However, as the standing wave is periodic in $d_{h k l}$, atomic positions at $m d_{h k l}$, where $m$ is an integer, are indistinguishable, so other information such as known atomic sizes and bond lengths have to be used to determine distances greater than $d_{h k l}$. One way to increase $d_{h k l}$ is to use synthetic layered materials and this has been successfully applied. ${ }^{9,10}$

An alternative way to form an X-ray standing wave is to reflect X-rays from a polished surface such that the incident and reflected waves overlap above the surface 11,17-25. To achieve a reflected X-ray of sufficient intensity to form the standing wave, the angle of incidence, $\alpha$, has to be in the region of the critical angle, $\alpha_{\text {crit }}$, of the surface. By scanning the incident angle from 0 to a few times $\alpha_{c r i t}$, an X-ray standing wave is produced above the surface which starts with an intensity period of $\infty$, but then falls to a period of $\approx 100 \AA$ at $\alpha_{\text {crit }}$ and continues decreasing for larger angles. The period is therefore variable, depending on the angle of incidence. Such variable period X-ray standing wave studies, also known as grazing incidence X-ray standing waves (GI-XSW), were first realised in $1989^{17}$ and can probe thicknesses of 10$100 \mathrm{~nm}$ with a spatial resolution of $\approx 0.2 \mathrm{~nm}$, which is $\approx 10$ to 100 times the thickness accessible to diffraction based XSW. 
Experiments have been carried out since the mid '70s to explore the possibilities of combining photoelectron spectroscopy and grazing incidence reflection. Early work by Mehta and Fadley ${ }^{26,27}$ established that the enhanced electric field at grazing incidence increased the relative sensitivity of surface species in XPS. Kawai et $\mathrm{al}^{28}$ carried out total reflection X-ray photoelectron spectroscopy (TRXPS) and presented a kinematical theory for the evanescent X-ray wave in the substrate surface region leading to enhanced surface sensitivity in XPS. This enhanced XPS surface sensitivity was further explored using $\mathrm{Si}$ samples ${ }^{29}$ and copper phthalocyanine on $\mathrm{Si}^{30}$ and in a review of TRXPS ${ }^{31}$ Kawai points out the possibilities of using X-ray standing waves monitored by photoelectron emission for thin film measurements. Chester et al used grazing incidence XPS (GIXPS) to enhance surface sensitivity of XPS and presented a dynamical X-ray theory to calculate X-ray intensities within the substrate and adlayers ${ }^{32,33}$. This was used to study relatively thin ( $\$ 50 \AA$ thick) gold films on Si surfaces, and oxide layers ( $\$ 70 \AA$ thick) on GaAs and it was noted that GIXPS would be a valuable tool for non-destructive depth profiling that is amenable to quantitation. Jach et al have gone on to apply GIXPS to thin oxide layers ( $\$ 75 \AA$ ) on $\mathrm{Si}^{34,3536}$ and also oxynitride layers ${ }^{37}(\leqslant 50 \AA)$ on $\mathrm{Si}$.

In this work we demonstrate that it is possible to quantitatively determine the structure of a thick film ( $\leq 250 \AA$ ) using several periods of an X-ray standing wave set up by reflection from a buried interface, in the manner of GI-XSW, using photoelectron detection rather than X-ray fluorescence to achieve chemical state specificity via the photoelectron chemical shift ${ }^{38}$. This eliminates the need for a crystalline substrate, greatly lengthens the analysis range and provides more structural information than the single period from Bragg diffraction. We demonstrate, using this technique, that the thickness of a thick molecular film composed of the light elements B, C, N, and F may be determined (211 $\pm 15 \mathrm{~A})$. We also show that it is possible to determine the position of a thin marker layer ( $12 \pm 10 \AA$ thick) containing other light elements $\mathrm{O}$ and $\mathrm{Cl}$ residing on the outside of the thick organic film. This work is a demonstration that VPXSW can be used as a viable structural tool for the analysis of thick films up to $250 \AA$ with chemical state specificity. 
To test and compare the spatial resolution and ability to detect light elements two model films were used. The first consisted of a silicon wafer covered by its inherent thin layer oxide surface. The second comprised a thick test film built in vacuum at 90 $\mathrm{K}$ on the oxide covered silicon wafer. It consisted of an organic "spacer" layer of ionic liquid, 1-octyl-3-methylimidazolium tetrafluoroborate, [OMIM][BF $]$, Fig. 1, grown to a depth of $\approx 200 \AA$ with a thin marker layer of chloroform $\left(\mathrm{CHCl}_{3}\right)$ and water, adsorbed on top of it. VPXSW experiments were carried out whilst monitoring the photoemission signals from Si, O, C, N, F, B and Cl. The results for the first sample, $\mathrm{Si} / \mathrm{SiO}_{2}$, found that the oxide layer was $20 \pm 10 \AA$ thick, with VPXSW data from silicon photoemission from the bulk and silicon and oxygen photoemission from the oxide, being fully consistent. Analysis of the marker layer showed the $\mathrm{CHCl}_{3}$ $\mathrm{H}_{2} \mathrm{O}$ layer to be $12 \pm 10 \AA$ thick, with its outer surface located $223 \pm 15 \AA$ from the surface, while analysis of the IL spacer layer signals showed a layer of IL $211 \pm 15 \AA$ thick with a marker layer $12 \pm 10 \AA$ thick (containing $\mathrm{CHCl}_{3}$ and $\mathrm{H}_{2} \mathrm{O}$ ) on top of it. Also VPXSW data obtained from silicon photoemission from the bulk and $\mathrm{SiO}_{2}$ layers below the organic layer were fitted with the same structural parameters as used for the marker and spacer layers, see Fig. 6B. This demonstrates that adlayers of the order of 100 's $\AA$ can be structurally analysed with an accuracy of 10-15 $\AA$.

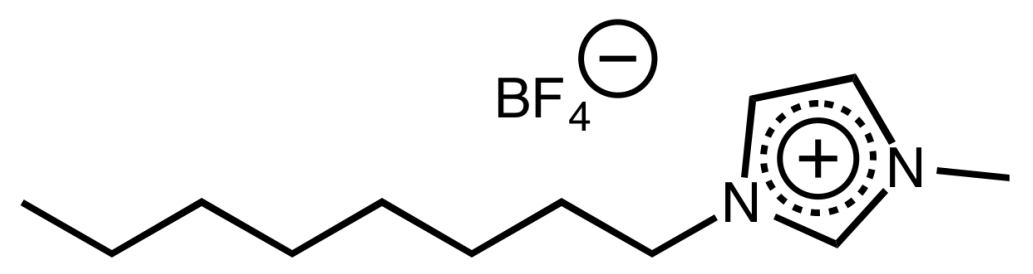

Figure 1. Structure of $[\mathrm{OMIM}]\left[\mathrm{BF}_{4}\right]$

\section{THEORY AND SIMULATIONS}

We have followed Zhang ${ }^{25}$ in calculating the X-ray amplitude and hence intensity for positions within the standing wave and hence within the adlayer. We use a three layer model where the vacuum is layer 1 , the adlayer, layer 2 , and the substrate layer 3 , Fig.2. The refractive index, $n$, of a medium for $\mathrm{X}$-rays is given by

$$
n=1-\delta-i \beta
$$

where $\beta$ is the absorption index and $\delta$ is the refractive index decrement. As $n$ is less than 1 , total external reflection occurs for incident angles, $\alpha$, smaller than the critical angle, $\alpha_{c}$, given to a good approximation by 


$$
\alpha_{c} \approx(2 \delta)^{1 / 2}
$$

For layer 1 (vacuum) the X-ray constants $\beta$ and $\delta$ are zero, and for layers 2 and 3 they were calculated ${ }^{39}$ for the material and the X-ray energy used $(3000 \mathrm{eV})$. For the first sample, the adlayer, layer 2 was $\mathrm{SiO}_{2}$ with $\beta_{2}=4.1625 \times 10^{-6}$ and $\delta_{2}=5.174 \times 10^{-5}$, giving a critical angle of $\alpha_{2, \text { crit }}=0.5828^{\circ}$ and the substrate, layer 3, was Si with $\beta_{3}=7.4498 \times 10^{-}$ ${ }^{6}$ and $\delta_{3}=5.4303 \times 10^{-5}$, giving a critical angle of $\alpha_{3, \text { crit }}=0.5971^{\circ}$. For the second sample the adlayer was primarily the ionic liquid with a marker layer consisting of $\mathrm{CHCl}_{3}$ and $\mathrm{H}_{2} \mathrm{O}$. We will refer to the $\mathrm{CHCl}_{3}-\mathrm{H}_{2} \mathrm{O}$-IL adlayer as the organic layer, sitting on the substrate, layer 3, which consisted of a thin layer of $\mathrm{SiO}_{2}$ on bulk $\mathrm{Si}$. For the purposes of calculating the X-ray intensity within the adlayer, the values of $\delta$ for the IL, $\mathrm{CHCl}_{3}$ and water for $3 \mathrm{keV} \mathrm{X}$-rays are sufficiently close, see A in the supplemental material ${ }^{40}$, that they can be considered to be the same. We have therefore used the values of the IL, $\beta_{2}=5.1271 \times 10^{-6}, \delta_{2}=2.7828 \times 10^{-5}$ giving $\alpha_{2, \text { crit }}=0.4274^{\circ}$. Similarly, for layer 3 , the $\mathrm{SiO}_{2}$ layer is thin and its $\delta$ value is sufficiently similar to $\mathrm{Si}$, and both are sufficiently different to the organic values, that the Si values were used for layer 3; see $\mathrm{A}$ in the supplemental material ${ }^{40}$ for more information. Using these constants and the angle of incidence, $\alpha$, the X-ray intensity can be calculated at any position, $z$, measured from the substrate/adlayer interface into the adlayer, as well as the intensity within the substrate layer and in the vacuum.

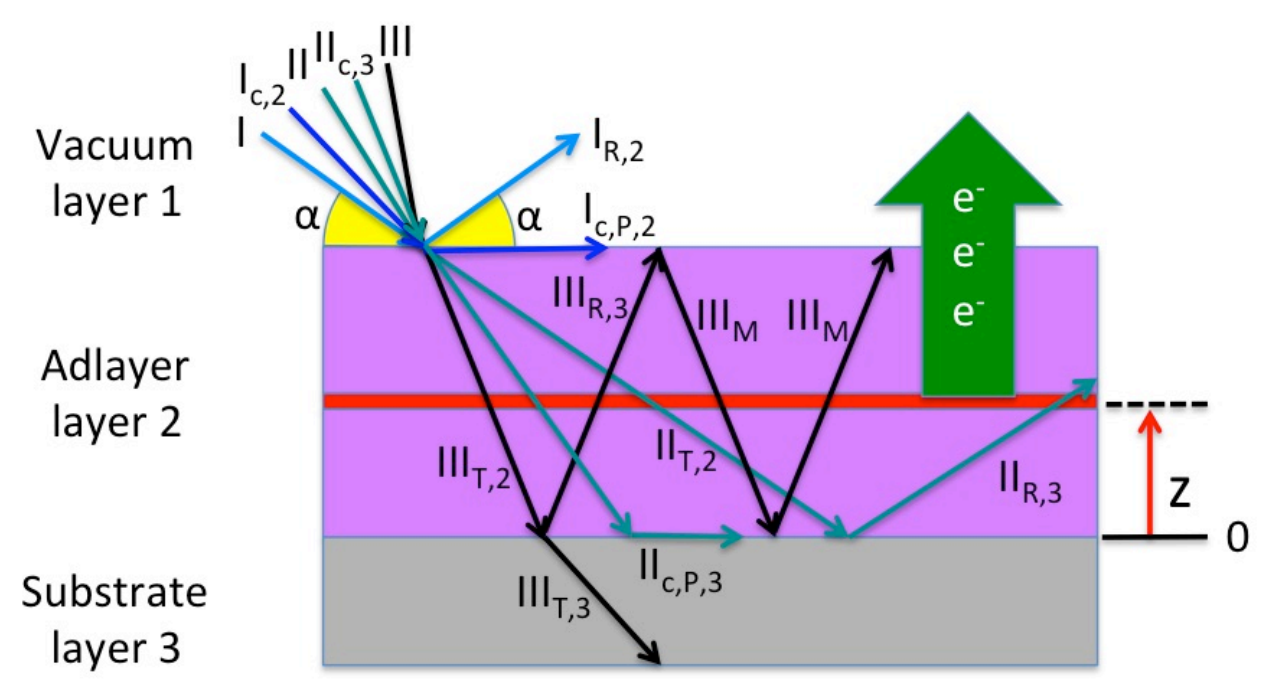

Figure 2. X-ray standing wave formation. Schematic of the behaviour of X-rays incident on a three layer surface comprising vacuum (layer 1), adlayer (layer 2) and substrate (layer 3 ) as the incident angle, $\alpha$, is increased from 0 . Various rays are 
shown (I, $\left.\mathrm{I}_{\mathrm{c}, 2}, \mathrm{II}, \mathrm{II}_{\mathrm{c}, 3}, \mathrm{III}\right)$ where $\mathrm{P}=$ parallel, $\mathrm{R}=$ reflected, $\mathrm{T}=$ transmitted, $\mathrm{M}=$ multiple reflections, $\mathrm{c}=$ critical angle and 1,2,3 refers to the higher layer number at an interface where $\mathrm{P}, \mathrm{R}$ or $\mathrm{T}$ occurs. A thin layer at position $z$ in layer 2 is shown emitting photoelectrons (large green arrow).

Figure 3A shows the X-ray intensity, normalised to the intensity of the incident wave, versus angle for positions $z$ along the surface normal for the simplest case where layers 1 and 2 are both vacuum above layer 3 which is a clean silicon surface. For increasing $\alpha$ the $\mathrm{X}$-ray intensity at $z=0$ rises to a maximum at the critical angle of the silicon, $\alpha_{3, \text { crit }}=0.597^{\circ}$, and then decays to an intensity corresponding to a travelling $\mathrm{X}$-ray wave propagating into the silicon bulk $(=1)$. Below the critical angle total external reflection occurs with a reflected wave above the surface and an evanescent wave propagating into the silicon. At the critical angle the reflected wave is at maximum intensity and a refracted wave forms, travelling along the silicon surface which causes the maximum in the standing wave intensity at $\mathrm{z}=0$. Above the critical angle a refracted wave propagates into the bulk while a reflected wave propagates into the vacuum. The incident and reflected waves combine above the surface to form a standing wave of intensity versus $z$ for any given $\alpha$. For a position $z=250 \AA$ out from the surface, five X-ray nodes and antinodes sweep past the position as $\alpha$ scans from $0^{\circ}$ to $2^{\circ}$, while for smaller value of $\mathrm{z}$ appropriately reduced modulations of X-ray intensity are experienced. Species at different distances $z$ will experience different standing waves, which is the "ruler" for this structural technique. For large angles the reflected wave intensity drops to zero causing the standing wave to disappear.

Figure 3B shows the effect on the X-ray intensity for a $250 \AA$ thick organic layer of the IL (layer 2) on Si (layer 3), Fig.2. For the ionic liquid the critical angle of the adlayer $\alpha_{c, \text { Org }}=0.427^{\circ}$. Hence for incident angles $0 \leq \alpha<\alpha_{c, \text { Org }} \mathrm{X}$-rays reflect from the top of layer $2(z=250 \AA)$, ray $\mathrm{I}_{\mathrm{R}, 2}$, Fig.2. The X-ray intensity at the layer $1 /$ layer 2 interface increases with increasing $\alpha$ while the intensity of the evanescent wave that penetrates below the interface, Fig. 3B, decreases as $\mathrm{z}$ decreases towards the bottom of layer 2 , such that there is almost no intensity at the layer $2 /$ layer 3 interface at $z=0$. For $\alpha=\alpha_{c, \text { Org }}\left(\right.$ ray $\mathrm{I}_{\mathrm{c}, 2}$ ) there is a reflected wave, and a transmitted wave (ray $\mathrm{I}_{\mathrm{c}, \mathrm{P}, 2}$ ) which is located at, and runs parallel to the layer 1/layer 2 interface, 
giving a maximum intensity at $z=250 \AA$. For incident angles that lie between the critical angles of the adlayer and the substrate, $\alpha_{c, \text { Org }}<\alpha<\alpha_{c, S i}$, Fig.2 II, the transmitted wave now propagates through layer 2 towards the layer 2/layer 3 interface (ray $\mathrm{II}_{\mathrm{T}, 2}$ ) where it reflects (ray $\mathrm{II}_{\mathrm{R}, 3}$ ) from layer 3 (silicon), for which the critical angle is $\alpha_{c, S i}=0.597^{\circ}$. The waves $\mathrm{II}_{\mathrm{T}, 2}$ and $\mathrm{II}_{\mathrm{R}, 3}$ form a standing wave within the adlayer, with an evanescent wave penetrating a short distance into the silicon. When $\alpha=\alpha_{c, S i}$, ray $\mathrm{II}_{\mathrm{c}, 3}$, another transmitted wave is formed at, and running parallel to, the layer 2/layer 3 interface, ray $\mathrm{II}_{\mathrm{c}, \mathrm{P}, 3}$, giving a maximum intensity at $z=0$, Fig.3B. For $\alpha>$ $\alpha_{c, S i} \mathrm{X}$-rays are partially reflected (ray $\mathrm{III}_{\mathrm{R}, 3}$ ) and partially transmitted, (ray $\mathrm{III}_{\mathrm{T}, 3}$ ) by the substrate, the two waves, $\mathrm{III}_{\mathrm{T}, 2}$ and $\mathrm{III}_{\mathrm{R}, 3}$, continuing to form a standing wave within layer 2. Eventually, as $\alpha$ increases the reflected wave decreases in intensity until there is simply a travelling wave passing through the entire surface. As can be seen from Fig. 3B, at any particular value of $\alpha>\alpha_{c, S i}$ there is an X-ray standing wave intensity with a period and an intensity that decreases as $\alpha$ increases. Multiple reflections within the adlayer (rays $\mathrm{III}_{\mathrm{M}}$ in Fig.2) modify the intensity of the standing wave substantially, causing higher and lower intensities than those found above the clean surface. The X-ray intensity within layer 3, the silicon substrate, can also be calculated and depends on the nature of layer 2 above it. Hence for quantitative results, the surface should be considered as a whole when calculating the standing wave. 

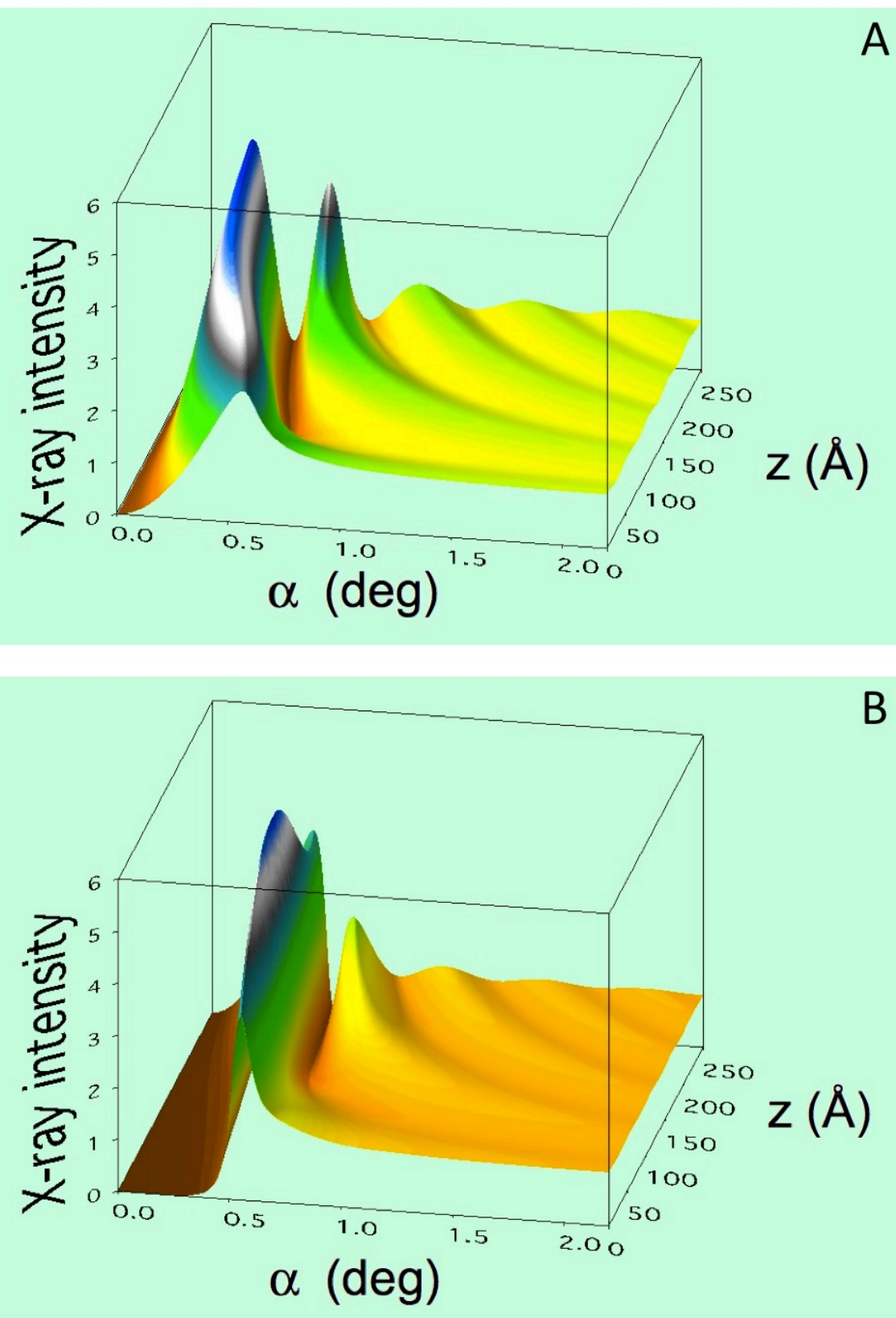

Figure 3. Calculated X-ray intensity versus angle $\alpha$ and position $z$ within layer 2 at an $\mathrm{X}$-ray energy of $3000 \mathrm{eV}$. A, for a clean Si surface where layer 2 is vacuum. B, for a $250 \AA$ A thick slab of [OMIM] $\left[\mathrm{BF}_{4}\right]$, layer 2, on a silicon substrate, layer 3.

$\mathrm{X}$-ray absorption at position $z$ is proportional to the X-ray intensity, and to the number density of absorbing species, at that position. Hence the photoelectron intensity 
generated at position $z$, and the hole density, will be proportional to X-ray intensity and the number density of the emitting species. Also the X-ray fluorescence intensity and Auger electron intensity generated from hole decay, travelling towards a detector are proportional to the X-ray intensity and number density of emitting species at $z$. The intensity of these emissions emerging from the surface of layer 2 , is given by the Beer-Lambert law which for photoelectrons travelling along the surface normal can be written

$$
I_{e} / I_{e 0}=\exp \left(-\left(d_{2}-z\right) / \lambda\right)
$$

where $I_{e 0}$ is the photoelectron intensity at position $z$ (valid for $0<\mathrm{z}<d_{2}$ ) travelling towards the analyser, $I_{e}$ is the photoelectron flux emerging into vacuum, $d_{2}$ is the thickness of layer 2 and $\lambda$ is the attenuation length for the particular electron kinetic energy of the emission travelling through layer 2. The measurable quantity is $I_{\text {exp }}$, the integral of $I_{e}$ over the distribution of species (from position $z_{1}$ to $z_{2}$ ) within the adlayer,

$$
I_{\text {exp }}=\int_{z_{1}}^{z_{2}} I_{e} d z
$$

To simulate the VPXSW curves, the substrate, layer 3 was considered to be of uniform density having X-ray constants $\beta_{3}$ and $\delta_{3}$. The adlayer, layer 2, was of uniform density, of thickness $d_{2}$ with X-ray constants $\beta_{2}$ and $\delta_{2}$. Photoelectron emission towards the surface, along the surface normal, was allowed to occur from a photoelectron region within layer 2 , having a relative density $\rho(z)$ of photoelectron emitting species, with its centre at position $z_{0}$ from the substrate surface, and of thickness $t$ i.e. from $z_{0}-t / 2$ to $z_{0}+t / 2$. The photoelectron region can be either a marker layer, a spacer layer, or the whole of layer 2, depending on the values of $z_{0}$ and $t$. At each incident angle $\alpha$, the intensity of the X-ray standing wave, $I(z)$, was calculated at each position $z$ through the photoelectron region. The photoelectron intensity for a given species at position $z$ was then calculated using

$$
I_{e 0}(z) \propto \rho(z) I(z) \delta z
$$

where $\delta z$ is the thickness element; in this work $\delta z=1 \AA$. The photoelectron flux, $I_{e}(z)$, from $\delta z$ emerging at the surface is given by equation (6) written explicitly with $I_{e}$ and $I_{e 0}$ as functions of $z$

$$
I_{e}(z)=I_{e 0}(z) \exp \left(-\left(d_{2}-z\right) / \lambda\right)(6)
$$


where $\lambda$ is the attenuation length for the photoelectron kinetic energy and the material comprising layer 2 . The attenuation length, $\lambda$, was taken to be $0.8 \lambda_{\text {imfp }}$ where $\lambda_{\text {imfp }}$ is the calculated inelastic mean free path for a particular photoelectron kinetic energy through the $\mathrm{IL}^{41}{ }^{42}$. The measured photoelectron flux leaving the surface along the surface normal for a particular angle of incidence, equation 4 , is then the sum of all contributions from $z$ across the thickness $t$ within the adlayer using increments $\delta z$,

$$
I_{\text {exp }}=\sum_{z_{0}-t / 2}^{z_{0}+t / 2} I_{e}(z) \delta z
$$

The calculation is repeated over the range of $\alpha$ used in the experiment.

To compare the simulated and experimental VPXSW data two corrections are necessary. Firstly, the simulation is corrected for the increased path length of the Xrays through the adlayer for small values of $\alpha$. This is achieved by division by $\sin \alpha$ (or by just $\alpha$, as the angles are small). Secondly, the calculated photoelectron intensity is corrected for the overlap of the X-ray footprint on the sample surface with the analysis area seen by the electron energy analyser. When the X-ray footprint is completely within the analyser field of view, all photoelectron intensity is captured and no correction is required. But for very low angles, the footprint falls outside the analyser field of view and signal is lost. The ratio of the X-ray beam diameter $\left(d_{\text {beam }}\right)$ to the concentric hemisphere analyser (CHA) field of view diameter $\left(d_{\text {anal }}\right)$ is used to determine the angle, $\alpha_{F P}$, at which the X-ray footprint starts to exceed the analyser field of view $\left(\sin \alpha_{F P}=d_{\text {beam }} / d_{\text {anal }}\right)$. In this work a ratio of 0.012 was used. The correction involves mutiplying the simulation intensity by $\sin \alpha_{F P} /\left(d_{\text {beam }} / d_{\text {anal }}\right)$, i.e. 1 , for $\alpha \geq \alpha_{F P}$ and by $\sin \alpha /\left(d_{\text {beam }} / d_{\text {anal }}\right)$ for $\alpha<\alpha_{F P}$. The footprint correction has the effect (after normalising to the maximum value in the simulated VPXSW scan to 1) of reducing the photoelectron intensity at higher angles, and is used to match the simulation and experimental intensities. The abrupt change at $\alpha_{F P}$ can lead to a noticeable kink in the simulation (see Fig.14), which could be smoothed if necessary. For $3000 \mathrm{eV} \mathrm{X}$-rays and $\alpha>0.1^{\circ}$ there is no appreciable attenuation of the X-rays as they pass through the adlayers used here, hence no correction for X-ray attenuation within the adlayer has been applied. The program used to calculate the VPXSW profiles is available from the Authors ${ }^{43}$. 

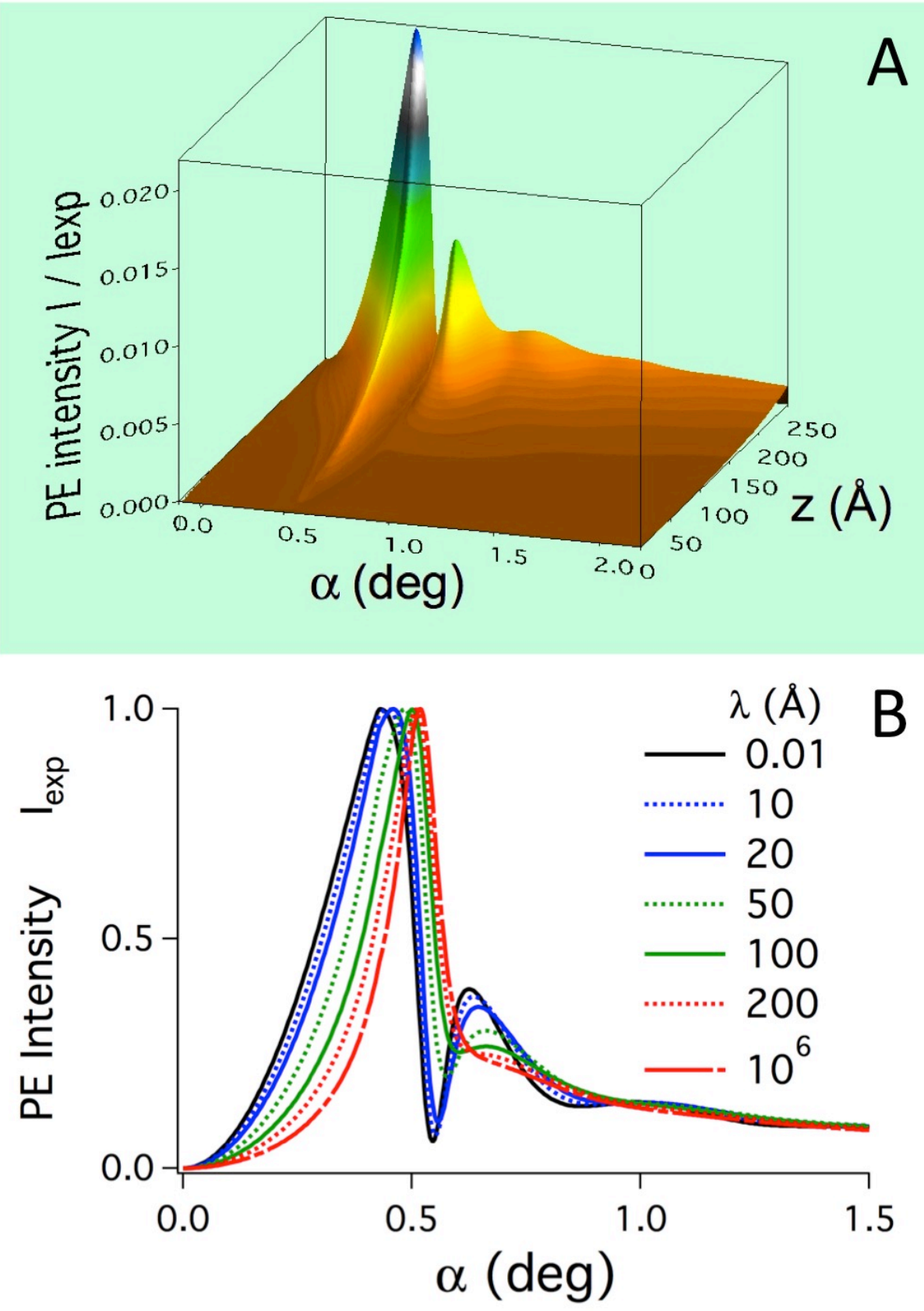

Figure 4. A, fraction of photoelectron intensity, $I_{e} / I_{\text {exp }}$, emerging from the surface of layer 2, which originates from a $1 \AA$ thick layer at position $z$, versus angle $\alpha$ calculated using $\lambda=50 \AA$. B, photoelectron intensity, $I_{\text {exp }}$, (normalised to 1 at the maximum) versus $\alpha$ plots for different values of the electron attenuation length $\lambda$. 
The $\lambda=10^{6} \AA$ curve is equivalent to X-ray fluorescent detection and is the same as a plot of the total hole density. Layer 2 is $[\mathrm{OMIM}]\left[\mathrm{BF}_{4}\right]$ with $d_{2}=250 \AA$, layer 3 is $\mathrm{Si}$, and the X-ray energy was $3000 \mathrm{eV}$. All plots are normalised to 1 at their maximum and have been corrected for the X-ray footprint and path length.

Figure 4A shows $I_{e} / I_{\text {exp }}$, the photoelectron intensity originating from a depth $z$ as a fraction of the experimentally determined signal versus $\alpha$ and $z$ for an IL slab with $d_{2}$ $=250 \AA$ using $\lambda=50 \AA$. This illustrates how different depths within the adlayer contribute to the experimental signal, depths less than $50 \AA$ contributing the most, while for depths $>250 \AA$ the signal drops to $<1 \%$. Figure 4B shows total photoelectron intensity, $I_{\text {exp }}$, expected from an homogenous slab of IL $250 \AA$ thick (layer 2) on Si for different values of the electron attenuation length $\lambda$, each normalised to 1 for its maximum value. For large values of $\lambda\left(=10^{6} \AA\right)$ the intensity is not attenuated by its passage through layer 2 and the plot is the same as for X-ray fluorescence detection, the signal being proportional to the sum of the hole densities within the slab caused by X-ray absorption. As $\lambda$ is reduced the photoelectron signal becomes increasingly dominated by a reduced thickness of material at the IL/vacuum interface. This causes the leading edge to move to lower angle and for oscillations to grow above $0.5^{\circ}$, due to the material becoming sensitive to the structure of the X-ray standing wave within the surface region of the slab. For $\lambda \leq 10 \AA$ the curve tends to a limit where only the top-most part of the slab contributes to the photoelectron signal. Clearly electron detection gives a more modulated signal than X-ray fluorescence (hole density) due to the averaging effect of a large $\lambda$ on the latter.

Figure 5A shows the total photoelectron signal, $I_{\text {exp }}$, versus $\alpha$ for slabs of [OMIM] $\left[\mathrm{BF}_{4}\right]$ of total thickness, $d_{2}$, on top of silicon. The thinnest slab of $10 \AA$ has a peak at $0.53^{\circ}$, slightly below the critical angle of $\operatorname{Si}\left(\alpha_{S i, c r i t}=0.5971^{\circ}\right)$. As the thickness increases the peak moves to lower angle, the drop on the high angle side becomes steeper and at $d_{2}=200 \AA$ a second peak develops at $0.74^{\circ}$. As the thickness increases further the second peak becomes more intense and moves to lower angles while the first peak continues shifting towards the critical angle of the IL $\left(\alpha_{\text {org, crit }}=\right.$ 
$0.4274^{\circ}$ ). Only minor changes occur in the leading edge. This behaviour may be interpreted as the X-rays penetrating the thin slab and reflecting from the IL/Si interface at the critical angle of the Si, causing the peak. As the slab gets thicker more reflection occurs from the vacuum/IL adlayer interface causing the peak to move towards the critical angle of the IL. For greater thickness two standing wave maxima sweep through the vacuum/IL interface causing the second peak at higher angle. Of relevance to the analysis below is that the plots for $230 \AA$ and $250 \AA$ are clearly distinguishable, the limit of distinguishability in the region of the second peak being about $\pm 10 \AA$.

Figure 5B shows $I_{\exp }$ which originates from thin markers layers of $t=2 \AA$ thickness centred at position $z_{0}$ within an IL adlayer of total thickness $d_{2}=250 \AA$. For the marker layer at $z_{0}=1 \AA$ there is a single peak at just below the critical angle of the Si. As the marker layer moves away from the IL/Si interface, for increasing $z_{0}$, the peak moves to lower angles, as it encounters the maximum in the X-ray standing wave further away from the Si surface at smaller angles. Simultaneously the leading edge extends to lower angles as the marker layer encounters more of the evanescent wave propagating down from the IL/vacuum surface. At $z_{0}=199 \AA$ a second peak appears at $0.805^{\circ}$, due to the marker layer being sufficiently far out that a second maximum in X-ray intensity can sweep through its position. As the $z_{0}$ position increases further toward the top of the IL layer this second peak moves to smaller angles, and for $\mathrm{z}_{0}=$ $230 \AA$ a third peak appears at $1.05^{\circ}$ where the third antinode of the standing wave passes through the marker layer. The curves for the marker layers are more modulated than those of the thick slabs. Comparing the 229 and $249 \AA$ curves, the position of thin marker layers should be locatable to $\pm 5 \AA$.

To determine the sensitivity to layer thickness, Fig.5C shows $I_{\exp }$ versus $\alpha$ for increasing thicknesses of marker layers at positions $z_{0} \pm t / 2$ within the $d_{2}=250 \AA \mathrm{slab}$ of IL. The marker layers all terminate at the IL/vacuum interface and become thicker towards the silicon surface. As the data is dominated by the material closest to the vacuum all the curves are similar. For thin layers $(248 \pm 1 \AA$ and $240 \pm 10 \AA)$ the error in thickness is about $\pm 10 \AA$, while for thick layers $(200 \pm 50$ and $125 \pm 125)$ it is about $\pm 35 \AA$. 


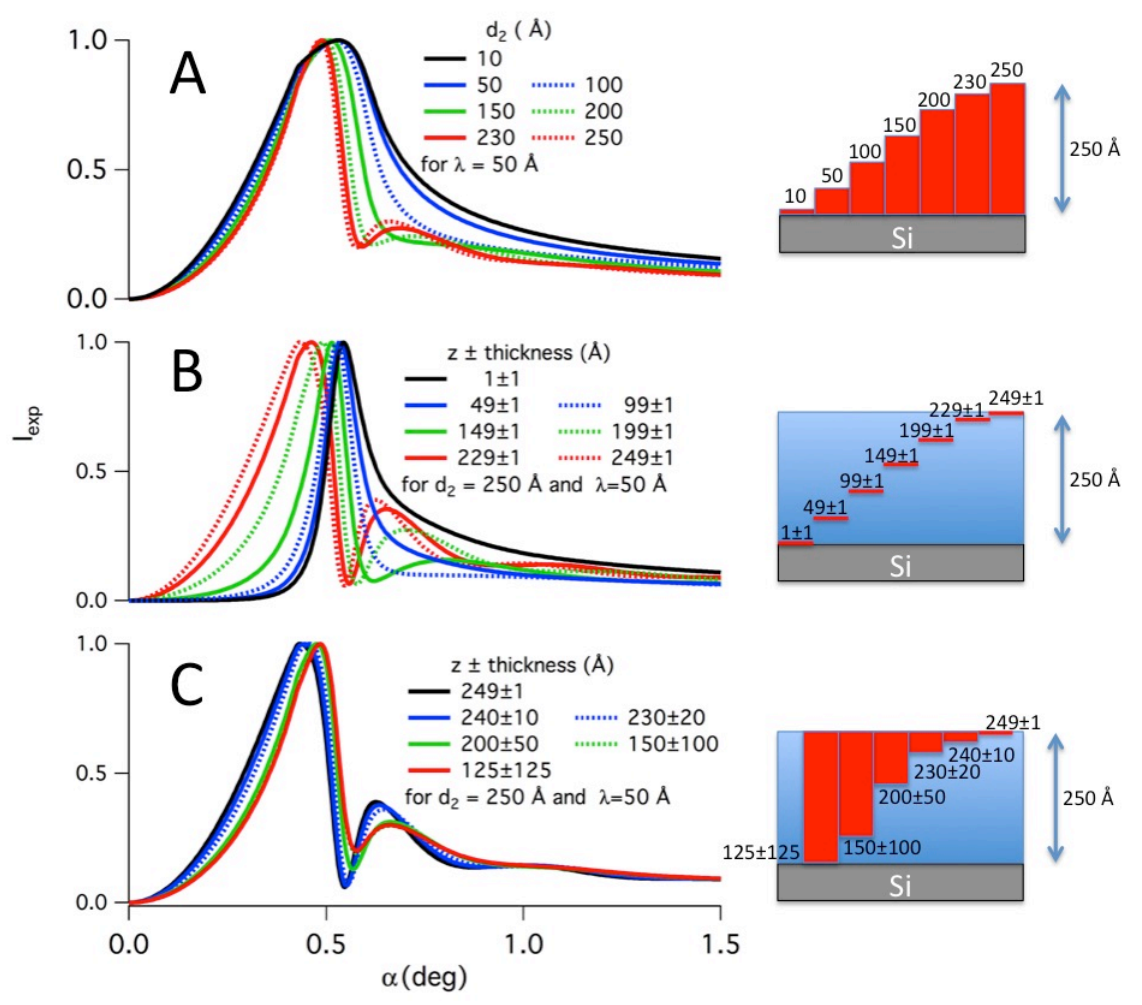

Figure 5. Normalised photoelectron intensity $I_{\exp }$ versus $\alpha$ for slabs of ionic liquid of thickness $d_{2}$ containing marker layers at position $z_{0}$ with a thickness either side of $z_{0}$ of $\pm \mathrm{t} / 2$, on top of silicon, using $3000 \mathrm{eV}$ X-rays and $\lambda=50 \AA$. A, $I_{\text {exp }}$ for a slab of thickness $d_{2}$. B, $I_{\text {exp }}$ from a marker layer $t=2 \AA$ thick $\left(z_{0} \pm 1 \AA\right)$ at position $z_{0}$ within an IL slab of thickness $250 \AA$. C, $I_{\exp }$ from marker layers of thickness 2 to $250 \AA$ terminating at the IL/vacuum interface. Corrections for X-ray footprint and path length have been made.

\section{EXPERIMENTAL DETAILS}

Experiments were carried out on beamline I09 at the Diamond Light Source, UK. The first sample was a silicon substrate $(10 \times 10 \times 1 \mathrm{~mm})$ with a thin native oxide on the surface, Fig. 6A. It was cleaned by washing with acetone prior to insertion into the vacuum chamber and measurements. The second sample, Fig.6B was built on the first by depositing the ionic liquid [OMIM] $\left[\mathrm{BF}_{4}\right]$, Fig.1, from an evaporator operated at $533 \mathrm{~K}^{44,45}$, onto the silicon substrate at $90 \mathrm{~K}$, followed by deposition of the two marker layer materials, chloroform $\left(\mathrm{CHCl}_{3}\right)$ and water, by adsorption from the gas phase. The IL evaporator had been previously calibrated in units of monolayers of IL s ${ }^{-1}$, where 1 monolayer corresponds to a layer $\approx 10 \AA$ thick, using temperature programmed desorption from a gold surface ${ }^{45}$. Deposition of $\approx 200$ 
$\AA$ of the IL was followed by $5 \times 10^{-6}$ mbar s exposure of $\mathrm{CHCl}_{3}(\approx 10 \AA$ thick $)$, followed by an exposure of $\approx 1 \times 10^{-6}$ mbar s of $\mathrm{H}_{2} \mathrm{O}(\approx 10 \AA$ thick $)$ adventitiously adsorbed over 2 hours from the background gases $\left(\approx 10^{-10} \mathrm{mbar}\right)$.

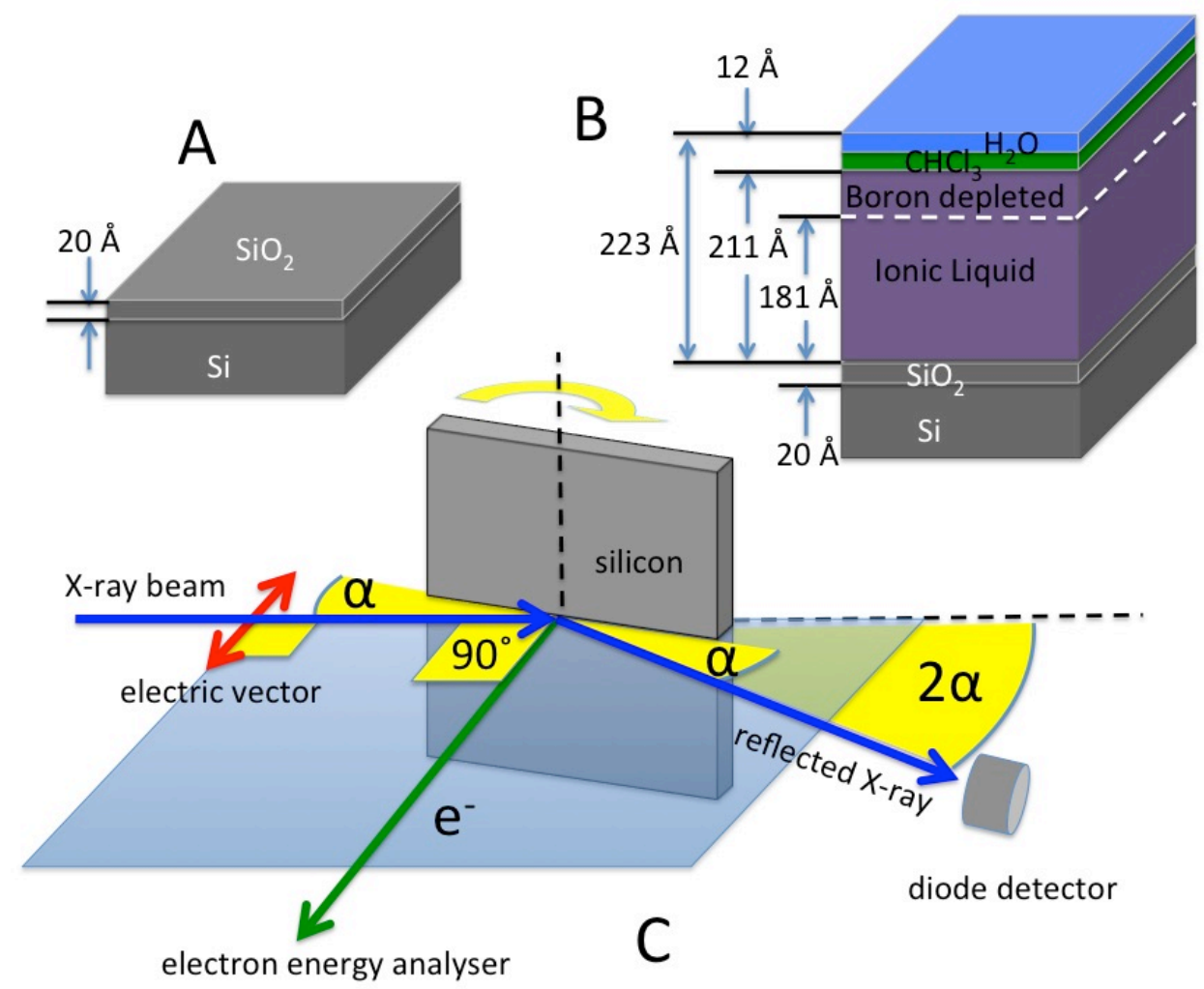

Figure 6. A, first sample, silicon with native silicon oxide on the surface. B, second sample, ionic liquid slab with thin marker layers of $\mathrm{CHCl}_{3}$ and adventitious $\mathrm{H}_{2} \mathrm{O}$. C, schematic showing the X-ray reflection and photoelectron analysis geometry. Layer thicknesses, as determined by VPXSW, are shown in A and B.

The X-ray beam was at $90^{\circ}$ to the electron energy analysis direction, and polarised with the electric field parallel to that direction, see Fig. 6C. This geometry minimises non-dipole photoemission effects ${ }^{46,47}$ on the photoelectron intensity. Variable period $\mathrm{X}$-ray standing wave experiments were carried out as follows. A $3000 \mathrm{eV}$ X-ray beam with dimensions $\approx 30 \mu \mathrm{m}$ (perpendicular to surface) $\times \approx 0.3 \mathrm{~mm}$ (parallel to surface) impinged at an angle $\alpha$ to the surface. This energy was chosen as it produces high KE photoelectrons $(\approx 2500 \mathrm{eV})$ which are penetrating $(\lambda \approx 55 \AA)$ but still have a photoemission cross-section large enough to make the peaks easily detectable. To minimise X-ray beam damage the intensity of the X-ray beam was attenuated by $200 \times$ 
using slits in the beamline $(20 \times)$ and by detuning the undulator gap $(10 \times)$. The energy distribution curves, (EDCs), of the electrons leaving the surface were measured with a concentric hemispherical analyser (VG Scienta EW4000 $10 \mathrm{keV}$, $200 \mathrm{~mm}$ radius hemisphere, lens acceptance angle $\pm 30^{\circ}$, resolution $<40 \mathrm{meV}$ at 3000 $\mathrm{eV}$ ). The CHA was operated with a pass energy of $100 \mathrm{eV}$ and accepted electrons from a field of view of $\approx 2.5 \mathrm{~mm}$ diameter, giving a ratio of 0.012 for the $\mathrm{X}$-ray beam size perpendicular to the surface divided by the diameter of the CHA field of view. This value is required to correct for the footprint of the X-ray beam falling outside the CHA field of view when comparing experimental and theoretical VPXSW curves. VPXSW scans were taken by scanning the incident angle $\alpha$ over the range $0-2.5^{\circ}$ in steps of $0.025^{\circ}$, with EDCs measured at each step. Note that this angular range changes the photoelectron path length through the adlayer by $<0.1 \%$ (emission angle of $90^{\circ}-87.5^{\circ}$ ), hence no correction for angular dependent attenuation of photoelectrons is required. For the first sample, clean silicon covered with native oxide, VPXSW scans were taken across the Si $2 \mathrm{p}$ and $\mathrm{O} 1 \mathrm{~s}$ photoelectron peaks at $293 \mathrm{~K}$. For the second sample, $\mathrm{Si} / \mathrm{SiO}_{2}$ substrate with IL spacer layer and a $\mathrm{CHCl}_{3} / \mathrm{H}_{2} \mathrm{O}$ marker layer on top, VPXSW scans were taken at $90 \mathrm{~K}$ across the Si $2 \mathrm{p}$, $\mathrm{Cl} 1 \mathrm{~s}, \mathrm{C} 1 \mathrm{~s}, \mathrm{~N} 1 \mathrm{~s}, \mathrm{O} 1 \mathrm{~s}, \mathrm{~F} 1 \mathrm{~s}$ and B 1s photoelectron peaks (15-30 mins duration for each). To further minimise beam damage the sample was moved $0.5 \mathrm{~mm}$ parallel to the surface to expose fresh surface for each VPXSW scan. Even with these precautions, beam damage was observed within the adlayer.

The photoelectron peaks within each EDC were fitted using Gaussian peaks (fitting parameters: position as binding energy $(\mathrm{BE} / \mathrm{eV})$, width (standard deviation, $\sigma / \mathrm{eV}$ ) and intensity (height or area) ) on a flexible polynomial background (up to 5th order). All fitting parameters could be automatically adjusted to accommodate charging and changing chemical environments during each VPXSW scan, ${ }^{48}$. Photoelectron peak areas, or sums of peak areas where appropriate, were then used to construct the VPXSW scans of photoelectron intensity versus angle.

\section{RESULTS AND DISCUSSION}

\section{A. First sample, silicon substrate with native oxide.}


Figure 7 shows the photoelectron (PE) spectrum of the $\mathrm{Si} / \mathrm{SiO}_{2}$ surface at $\alpha=2.5^{\circ}$.

The $2 \mathrm{p}_{3 / 2}$ and $2 \mathrm{p}_{1 / 2}$ components of bulk $\mathrm{Si}$ and $\mathrm{Si}$ in $\mathrm{SiO}_{2}$ are at binding energies (BEs) of $\approx 100 \mathrm{eV}$ and $\approx 103 \mathrm{eV}$, the latter broadened due to the range of chemical environments within the surface oxide. All four components, Fig.7A, were fitted using Gaussian line shapes, as was the single component for the $\mathrm{O} 1 \mathrm{~s}$ peak $(\mathrm{BE}=$ $532.3 \mathrm{eV}$ ), Fig.7B, from the $\mathrm{SiO}_{2}$ layer. Such fitting was carried out for all the PE spectra obtained over the incident angle range of $0-2.5^{\circ}$. The Si $2 p$ chemical shift between bulk and oxide components was $3.5 \mathrm{eV}$, slightly smaller but consistent with refs ${ }^{49,50}$ which have chemical shifts of 3.7 and $4.3 \mathrm{eV}$ for the unresolved $\mathrm{Si}_{3 / 2,1 / 2}$ between bulk $\mathrm{Si}$ and the $\mathrm{SiO}_{2}$. The fitting parameters, measured at $\alpha=2.5^{\circ}$, are listed in table 1 .
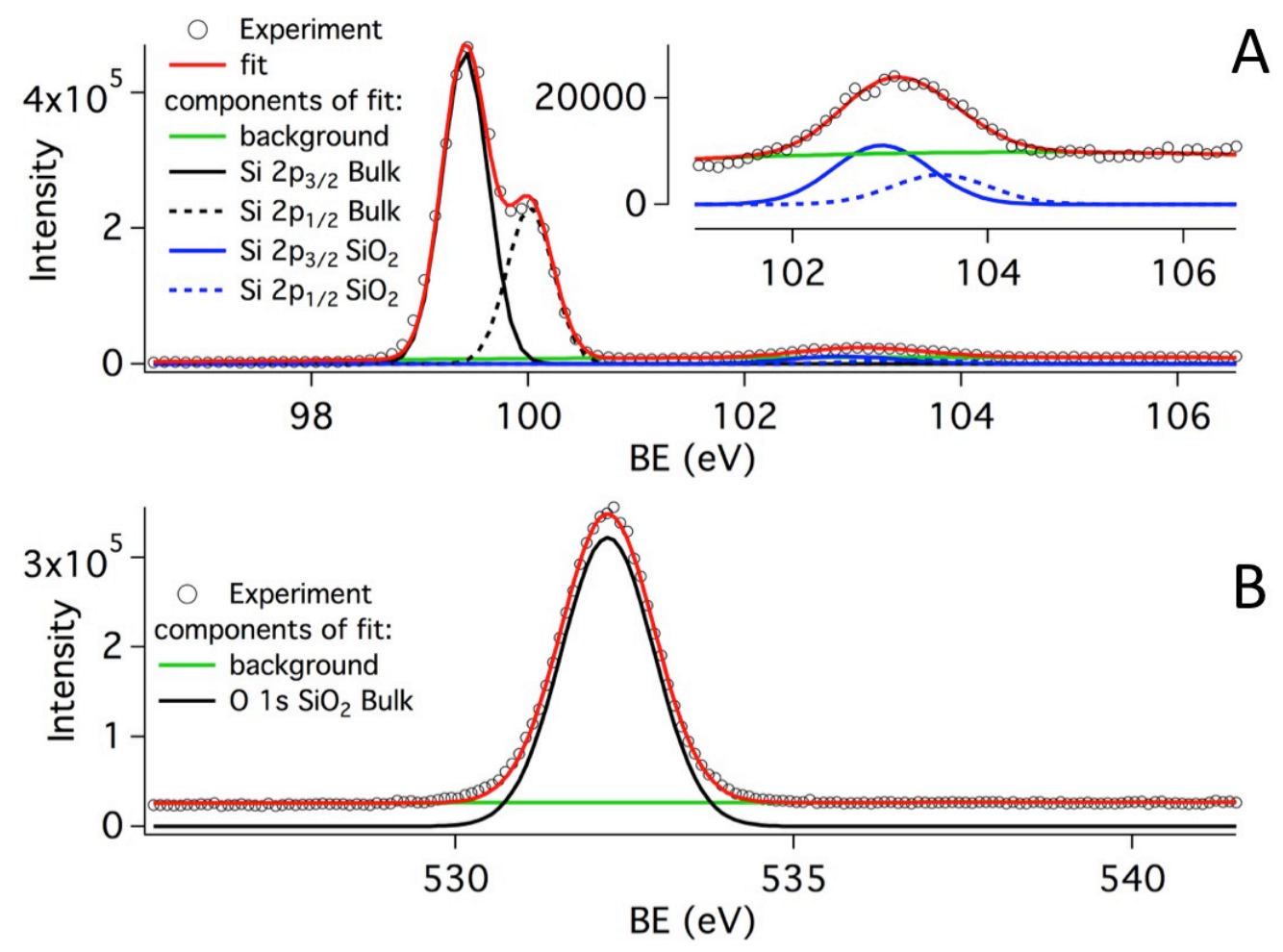

Figure 7. Photoelectron spectra from the native oxide covered silicon surface. A, Si $2 p$ fitted using a splitting of $0.6 \mathrm{eV}$ between $2 \mathrm{p}_{1 / 2}$ and $2 \mathrm{p}_{3 / 2}$, an intensity ratio of $2: 1$, and a chemical shift of $3.5 \mathrm{eV}$ for $\mathrm{SiO}_{2}$. Inset shows an expanded view of the $\mathrm{SiO}_{2}$ region. $\mathrm{B}, \mathrm{O} 1 \mathrm{~s}$ fitted with a single component. Taken at $\alpha=2.5^{\circ}$. 
Table 1. Photoelectron kinetic and binding energies and calculated attenuation lengths.

\begin{tabular}{|c|c|c|c|c|}
\hline Core level & $\begin{array}{c}\text { Binding } \\
\text { Energy / eV }\end{array}$ & $\begin{array}{l}\text { Photoelectron } \\
\text { kinetic energy } \\
\qquad / \mathrm{eV} \S\end{array}$ & $\begin{array}{c}\text { IMFP / } \AA \text { and } \lambda \\
/ \AA \\
51 *\end{array}$ & width $\sigma / \mathrm{eV} \dagger$ \\
\hline $\mathrm{Cl} 1 \mathrm{~s}_{\mathrm{CHCl} 3}$ & $2823.2(1)$ & \multirow{3}{*}{$\approx 177$} & \multirow{3}{*}{11,9} & $1.6(1)$ \\
\hline $\mathrm{Cl} 1 \mathrm{~s}_{\mathrm{Cl}-}$ & $2820.9(1)$ & & & $1.3(1)$ \\
\hline $\mathrm{Cl} 1 \mathrm{~s}_{\mathrm{Cl}} \bullet$ & $2825.6(1)$ & & & $1.6(1)$ \\
\hline $\mathrm{F} 1 \mathrm{~s}_{\text {anion }}$ & $686.3(1)$ & $\approx 2314$ & 61,49 & $1.4(1)$ \\
\hline $\mathrm{O} 1 \mathrm{~s}_{\mathrm{H} 2 \mathrm{O}}$ & $533.4(1)$ & \multirow{3}{*}{$\approx 2468$} & \multirow{3}{*}{64,51} & $1.4(1)$ \\
\hline $\mathrm{O} 1 \mathrm{~s}_{\text {damage }}$ & $531.2(1)$ & & & $1.0(1)$ \\
\hline $\mathrm{O} 1 \mathrm{~s}_{\mathrm{SiO} 2}$ & $532.3(1)$ & & & $1.0(1)$ \\
\hline $\mathrm{N} 1 \mathrm{~s}_{\text {cation }}$ & $401.6(1)$ & \multirow{2}{*}{$\approx 2598$} & \multirow{2}{*}{66,53} & $1.4(1)$ \\
\hline $\mathrm{N} 1 \mathrm{~s}_{\text {damage }}$ & $399.6(1)$ & & & \\
\hline C 1 $\mathrm{s}_{\text {alkyl }}$ & $284.9(1)$ & \multirow{2}{*}{$\approx 2715$} & \multirow{2}{*}{68,54} & $1.1(1)$ \\
\hline C 1 shetero & $286.5(1)$ & & & \\
\hline B 1s & 194.2(1) & $\approx 2806$ & 70,56 & $1.1(1)$ \\
\hline Si $2 p_{1 / 2 \text { bulk }}$ & $100.0(1)$ & \multirow{4}{*}{$\approx 2901$} & \multirow{4}{*}{72,58} & $0.3(1)$ \\
\hline Si $2 p_{3 / 2 \text { bulk }}$ & $99.4(1)$ & & & $0.3(1)$ \\
\hline $\mathrm{Si} 2 \mathrm{p}_{1 / 2 \mathrm{SiO} 2}$ & $103.5(1)$ & & & $0.7(1)$ \\
\hline Si $2 p_{3 / 2 \mathrm{SiO} 2}$ & $102.9(1)$ & & & $0.7(1)$ \\
\hline
\end{tabular}

* IMFP is the inelastic mean free path; $\lambda$ is the attenuation length $=0.8 \times$ IMFP. An average value of $\lambda=55 \AA$ was used for all photoelectrons except $\mathrm{Cl} 1 \mathrm{~s}$.

$\dagger \sigma$ is the standard deviation of the Gaussian peak used for fitting.

$\$$ Single point calibration using Si $2 \mathrm{p}_{3 / 2}=99.42 \mathrm{eV} \mathrm{BE}^{52}$. Measured at $\alpha=2.5^{\circ}$.

$\S$ Calculated using an X-ray energy of $3000 \mathrm{eV}$. 


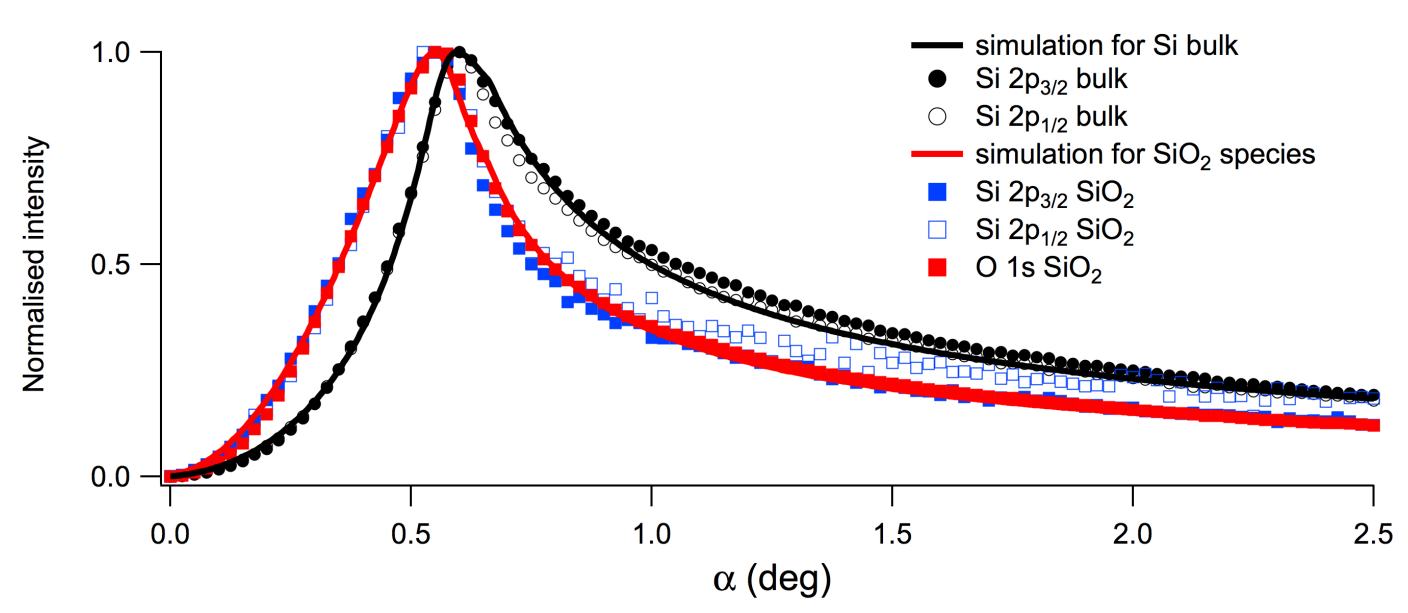

Figure 8. VPXSW scans of the $\mathrm{O} 1 \mathrm{~s}, \mathrm{Si} 2 \mathrm{p}_{1 / 2}$ and $\mathrm{Si} 2 \mathrm{p}_{3 / 2}$ for Si bulk and $\mathrm{SiO}_{2}$. The simulations (normalised to 1, corrected for footprint and path length and shifted by $0.025^{\circ}$ ) are for an oxide layer $20 \AA$ thick, see cartoon Fig. 6A.

The VPXSW scans of photoelectron intensities versus angle of incidence, normalised to a value of 1 at their maximum, are shown in Fig.8. The scans obtained using the Si $2 p_{3 / 2}$ and $2 p_{1 / 2}$ components of bulk Si are the same to within experimental error, as expected, with a peak at the critical angles of the $\mathrm{Si}$ and $\mathrm{SiO}_{2}$. The shapes of the scans of the two $\mathrm{Si}$ components and the $\mathrm{O}$ 1s component from $\mathrm{SiO}_{2}$ are also within error of each other, as expected if they sense the same standing wave within the oxide layer. The three curves from $\mathrm{SiO}_{2}$ have a leading edge that rises more quickly than the bulk curves, because they encounter the standing wave earlier (at lower $\alpha$ ). Figure 8 also shows a simulated VPXSW curve for the oxide species, corrected for the X-ray footprint and path length, for an oxide thickness of $20 \AA$ and $\lambda=58 \AA$ for Si $2 p$ photoelectron transmission through the $\mathrm{SiO}_{2}$. The fit is rather good over the entire range of $2.5^{\circ}$, indicating that the theory generally, and the correction for path length in particular, are correct. Also shown in Fig.8 is a simulated VPXSW curve for the bulk Si substrate, calculated using the same oxide thickness of $20 \AA$, and $\lambda=$ $58 \AA$ for photoelectron transmission through the bulk $\mathrm{Si}$ and $\mathrm{SiO}_{2}$ (using $\lambda=51 \AA$ for transmission of O1s photoelectrons through $\mathrm{SiO}_{2}$ has no significant effect on the data). The fit to the experimental data is again rather good. Both simulated curves have been shift by $0.025^{\circ}$ on the $\alpha$ scale to bring the theory and experimental angular ranges into coincidence. This represents one increment of $\alpha$ in the experiment and is the accuracy with which we can define $\alpha=0$ ). Following considerations described in 
the theory section, and more specifically in $\mathrm{C}$ in the supplemental material ${ }^{40}$, the error in the thickness is estimated as $\pm 10 \AA$.

\section{B. Second sample, $\mathrm{Si}-\mathrm{SiO}_{2} / \mathrm{IL}-\mathrm{CHCl}_{3}-\mathrm{H}_{2} \mathrm{O}$}

The C, N, F and B 1s photoelectron peaks from the ionic liquid spacer layer, taken at $\alpha=2.5^{\circ}$, are shown in Fig. 9 with best fit components, the parameters of which are shown in Table1. The $\mathrm{C}$ 1s shows two components due to the alkyl chain carbons, $\mathrm{C}_{\mathrm{alkyl}}$, and the carbons in the imidazolium region, $\mathrm{C}_{\text {hetero, }}{ }^{53,54}$. The carbon $1 \mathrm{~s}$ signal from the $\mathrm{CHCl}_{3}$ would be expected to have a binding energy of $\approx 289.7 \mathrm{eV}(\approx 4.8 \mathrm{eV}$ higher than the alkyl peak ${ }^{55}$ ) but the intensity is too low to allow it to be distinguished from the high binding side of imidazolium carbon signal.

The N 1s peak, Fig. 9B, exhibits two peaks, the higher binding energy peak corresponding to the imidazolium nitrogens in the cation, $\mathrm{N}_{\text {cat }}$, the smaller, lower BE peak to nitrogen in a beam damaged species, $\mathrm{N}_{\text {damage }}{ }^{56}$. Figures $9 \mathrm{C}$ and $9 \mathrm{D}$ show the fluorine and boron photoelectron peaks, respectively, each fitted with a single component. The two unfitted peaks at $\approx 198.5$ and $\approx 200.5 \mathrm{eV}$ are due to $\mathrm{Cl} 2 \mathrm{p}_{3 / 2}$ and $2 \mathrm{p}_{1 / 2}$ photoelectrons from the chloroform marker layer. As the nitrogen peak in the cation shows beam damage we expect the other peaks to also show damage. Although there are no clearly identifiable damage peaks in the $\mathrm{C} 1 \mathrm{~s}$ spectra, the $\mathrm{C}$ $1 \mathrm{~s}_{\text {hetero }}$ is particularly broad, table 1 , indicating there may be intensity changes due to damage within the envelope of the peak. The lack of any damage peaks in the F 1s and $\mathrm{B} 1 \mathrm{~s}$ is surprising but may be due to the formation of $\mathrm{BF}_{3}$ as a damage product, which desorbed and hence did not leave a peak for XPS to detect. This is discussed later and in appendix 2.

For the marker layer surface, the $\mathrm{Cl} 1 \mathrm{~s}$, Fig. 4B, showed three peaks in the EDC taken $\alpha=2.5^{\circ}$. The middle $\mathrm{BE}$ peak, $\mathrm{Cl} 1 \mathrm{~s}_{\mathrm{CHCl} 3}(2823.2 \mathrm{eV} \mathrm{BE})$ can be assigned to undamaged $\mathrm{CHCl}_{3}$ as it was the sole observable peak for EDCs taken at low X-ray exposure times, and it decreased with exposure to the beam. The higher intensity, lower BE peak, $\mathrm{Cl} 1 \mathrm{~s}_{\mathrm{Cl}}^{-},(2820.9 \mathrm{eV})$ we assign to a damage product (as it increased with exposure to beam). As the binding energy is lower than that of $\mathrm{CHCl}_{3}$ we suggest it is due to $\mathrm{Cl}^{-}$, caused by interaction of $\mathrm{CHCl}_{3}$ and the incident $\mathrm{X}$-rays and/or 
photo-generated electrons. The low intensity, higher BE component, $\mathrm{Cl} 1 \mathrm{~s}_{\mathrm{Cl}} \bullet$ $\mathrm{eV}$ ), we identify as due to approximately neutral chlorine, perhaps due to chlorine atoms, or $\mathrm{Cl}_{2}$ which had formed from chlorine atoms and was trapped or stabilised within the solid IL. The O 1s spectrum from water in the marker layer, Fig. 10B, showed one peak due to adsorbed water, $\mathrm{O} 1 \mathrm{~s}_{\mathrm{H} 2 \mathrm{O}}$, and a very small peak $\mathrm{O} 1 \mathrm{~S}_{\text {damage }}$ which became clearly visible at some angles, which we cannot further identify. The $\mathrm{O} 1 \mathrm{~s}_{\mathrm{SiO} 2}$ peak was not observed as it was too attenuated by the spacer layer to contribute to the spectrum. 


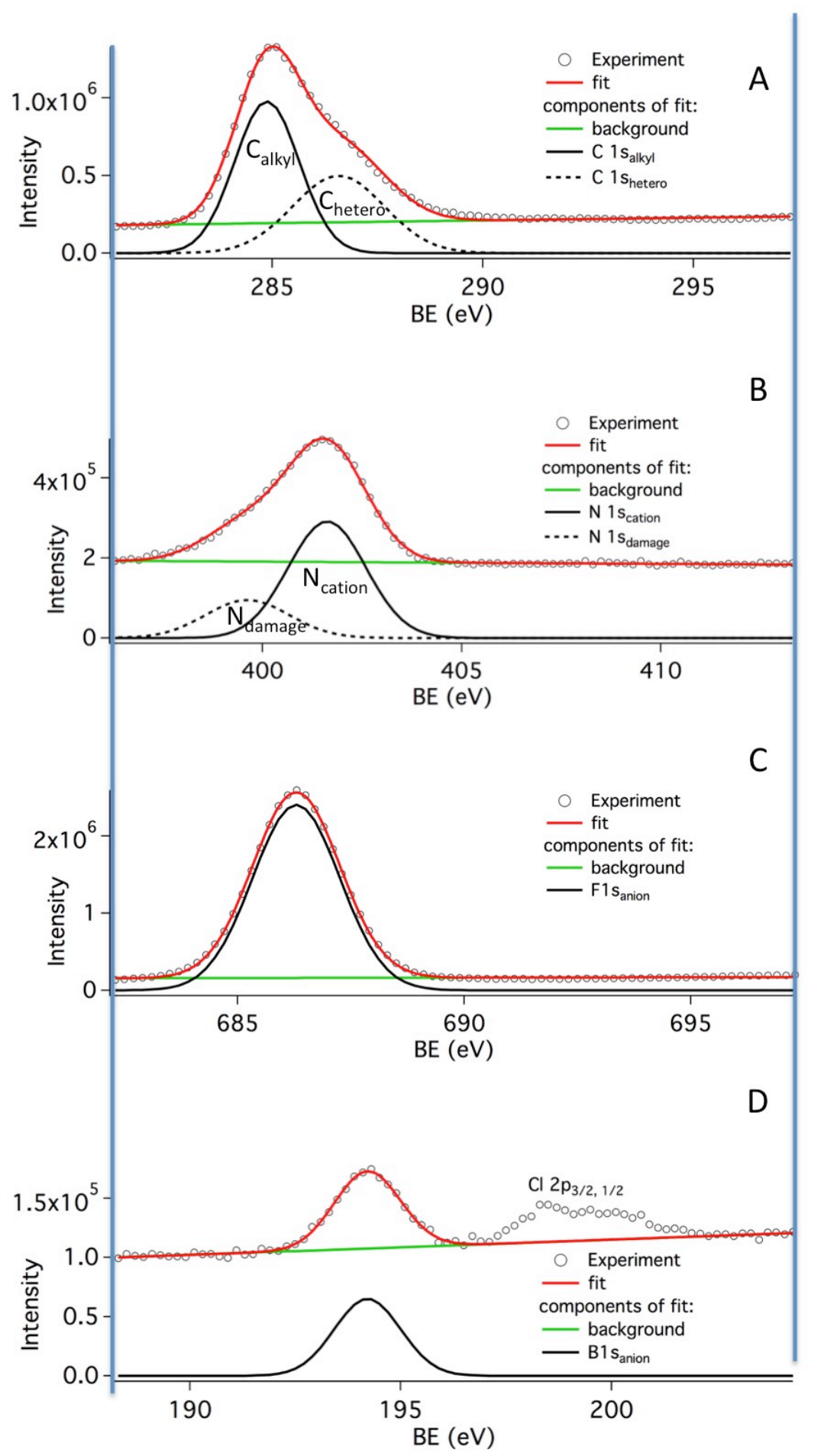

Figure 9. Photoelectron spectra of $[\mathrm{OMIM}]\left[\mathrm{BF}_{4}\right]$ from the spacer layer of the Second sample, $\mathrm{Si}-\mathrm{SiO}_{2} / \mathrm{IL}_{-} \mathrm{CHCl}_{3}-\mathrm{H}_{2} \mathrm{O}$, taken at $\alpha=2.5^{\circ}$. A, C $1 \mathrm{~s}$ fitted with two components $\mathrm{C} 1 \mathrm{~s}_{\text {alkyl }}$ and $\mathrm{C} 1 \mathrm{~s}_{\text {hetero. }} \mathrm{B}, \mathrm{N} 1 \mathrm{~s}$ fitted with two components, $\mathrm{N} 1 \mathrm{~s}_{\text {cat }}$ and $\mathrm{N} 1 \mathrm{~s}_{\text {damage. }} \mathrm{C}$, F 1s photoelectron spectrum fitted with a single component. D, B 1s photoelectron spectrum fitted with a single component. 

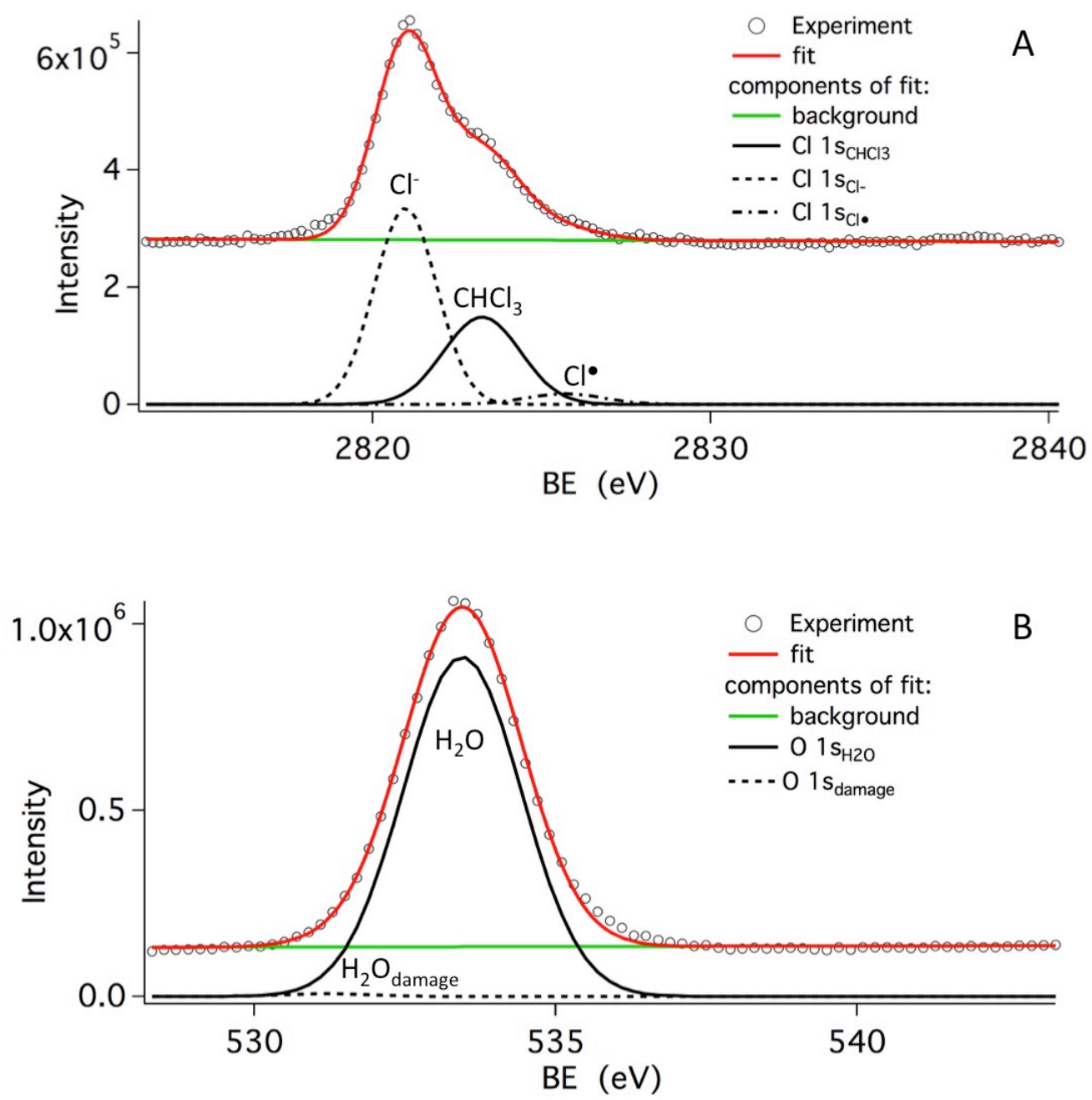

Figure 10. Photoelectron spectra of [OMIM] $\left[\mathrm{BF}_{4}\right]$ from the marker layer of the 2nd thin film sample, taken at $\alpha=2.5^{\circ}$. A, $\mathrm{Cl} 1 \mathrm{~s}$ fitted with three components, $\mathrm{Cl} 1 \mathrm{~s}_{\mathrm{CHCl} 3}$, $\mathrm{Cl} 1 \mathrm{~s}_{\mathrm{Cl}-}$ and $\mathrm{Cl} 1 \mathrm{~s}_{\mathrm{Cl}} \bullet . \mathrm{B}, \mathrm{O} 1 \mathrm{~s}$ fitted with two components $\mathrm{O} 1 \mathrm{~s}_{\mathrm{H} 2 \mathrm{O}}$ and $\mathrm{O} 1 \mathrm{~s}_{\text {damage. }}$ All fitting parameters are listed in table 1.

Figure 11A shows the VPXSW data for chlorine as EDCs stacked to form a 3D plot of electron intensity versus binding energy (BE) and $\alpha$. The same fitting procedure as described above was used but allowing the BEs and widths to change to accommodate peak movement. The $\mathrm{Cl} 1 \mathrm{~s}_{\mathrm{CHCl}}$ peak becomes visible at a $\mathrm{BE} \approx 2822.4 \mathrm{eV}$. As the angle of incidence increases, the peak increases in intensity and moves to higher BE. It then reverses direction and decreases in BE while the intensity continues to increase to a maximum (most easily seen in the contour plot of Fig. 11A). Figure 11B shows 
the $\mathrm{BE}$ and intensity of this peak after fitting. The BE maximises at a value $9.3 \mathrm{eV}$ higher than its starting value at $\alpha \approx 0.25^{\circ}$ while the intensity maximises at $\alpha \approx 0.42^{\circ}$, and both drop to a local minima at $\approx 0.55^{\circ}$ beyond which the BE only changes very slightly. This behaviour of the binding energy may be interpreted as follows. At 90 $\mathrm{K}$ the IL slab behaves as an insulator, as the ions cannot move to conduct current, and hence the adlayer is prone to charging by loss of electrons under the action of photoemission. The initial increase in BE is due to positive charging of the organic layer as the evanescent X-ray wave at the vacuum/organic layer interface penetrates further into the organic layer as $\alpha$ increases, causing greater photoemission of electrons. The maximum intensity of the $\mathrm{Cl} 1 \mathrm{~s}$ peak coincides with the critical angle of the vacuum/organic layer interface $\left(0.4274^{\circ}\right)$ where the refracted X-ray travels parallel to the surface and within the chloroform marker layer (ray $\mathrm{I}_{\mathrm{c}, \mathrm{P}, 2}$ in Fig.2), giving it a maximum path length, and hence maximum absorption and photoelectron intensity. The angle at which photoelectron intensity reaches a minimum, and the BE abruptly flattens is close to the critical angle of the organic $/ \mathrm{SiO}_{2}$ interface $\left(0.5828^{\circ}\right)$ where the refracted X-ray wave runs along the $\mathrm{IL} / \mathrm{SiO}_{2}$ interface and hence the intensity in the marker layer is greatly reduced. Interestingly, maximum charging as indicated by the maximum shift in binding energy, occurred at a significantly smaller angle than maximum photoemission. We would expect maximum charging to occur when the total electron flux leaving the sample is a maximum, which is not necessarily the same angle at which the $\mathrm{Cl} 1 \mathrm{~s}$ adsorbate photoelectron flux maximises. Unfortunately the drain current, which is the same as the total electron flux leaving the sample, was not monitored in this work so we are unable to verify whether the charging does maximize at the same angle at which emission of all electrons reaches a maximum. 

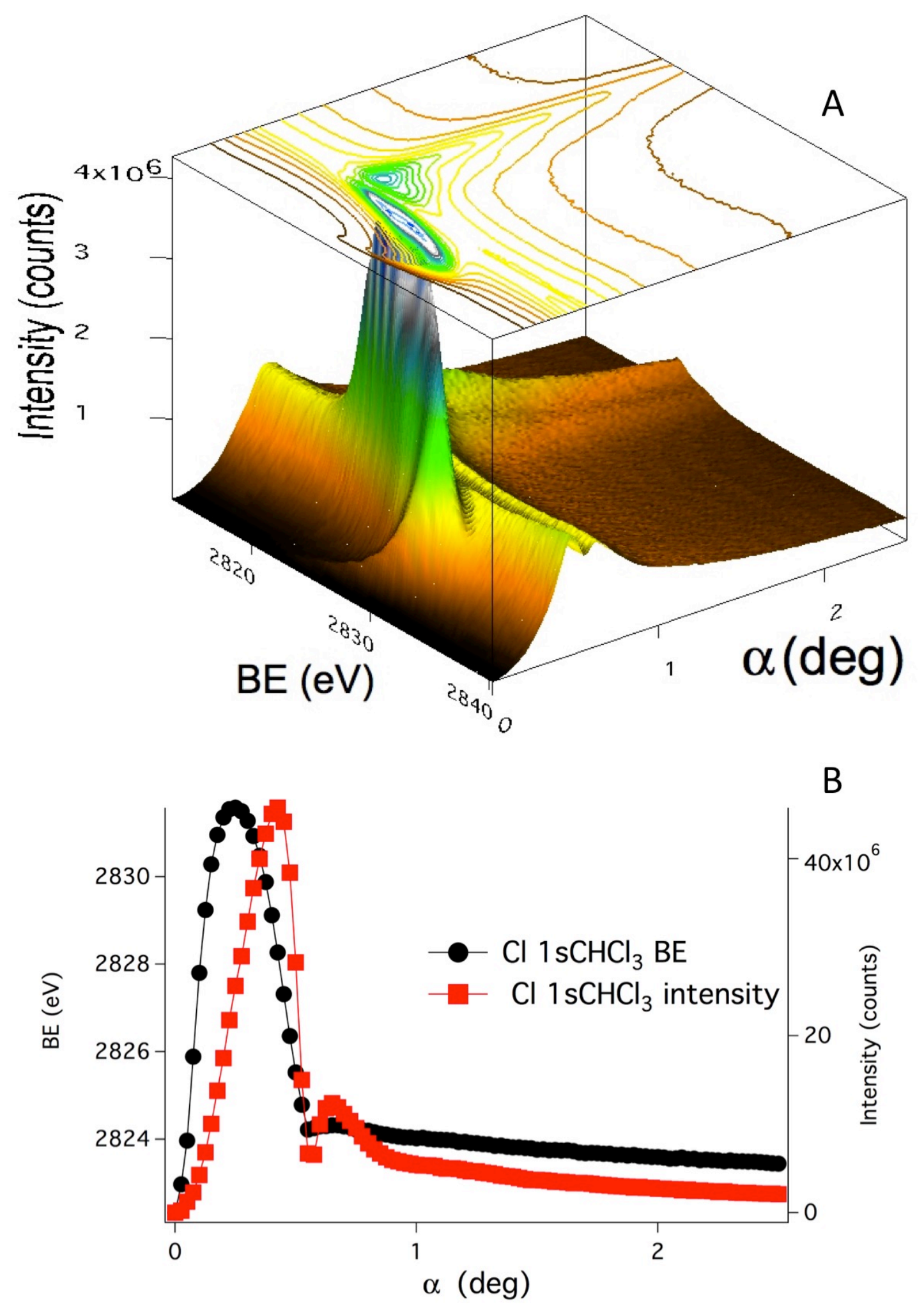

Figure 11. Experimental results for thin film of $\mathrm{CHCl}_{3} / \mathrm{H}_{2} \mathrm{O}$ on $\mathrm{IL}$ on $\mathrm{Si} / \mathrm{SiO}_{2}$. A, VPXSW data as $\mathrm{Cl} 1 \mathrm{~s}$ photoelectron intensity versus $\mathrm{BE}$ and $\alpha^{\circ}$ and as a contour plot. $\mathrm{B}, \mathrm{BE}$ and intensity of the $\mathrm{Cl} 1 \mathrm{~s}_{\mathrm{CHCl} 3}$ component after fitting the data in $\mathrm{A}$. 
The VPXSW scans of the chemically shifted chlorine components, $\mathrm{Cl} 1 \mathrm{~s}_{\mathrm{CHCl}}, \mathrm{Cl}$ $1 \mathrm{~s}_{\mathrm{Cl}}$ and $\mathrm{Cl} 1 \mathrm{~s}_{\mathrm{Cl}}$ are shown in Fig. $12 \mathrm{~A}$ as fitted intensity versus $\alpha$. The intensity of the $\mathrm{CHCl}_{3}$ component rises quickly to a maximum at 0.43 degrees and is more intense than the $\mathrm{Cl}^{-}$component. By $0.55^{\circ}$ they have the same intensity and to higher angles the $\mathrm{Cl}^{-}$is the more intense component (see Fig.10A for the components at $\alpha=2.5^{\circ}$ ). This is consistent with the chloroform cracking under the X-ray beam, but as the standing wave intensity is the primary source of the cracking, the major X-ray dose occurs over the range $0-0.55^{\circ}$ beyond which the X-ray intensity settles to much lower values. This means the major changes in the $\mathrm{CHCl}_{3}$ (decreasing) and $\mathrm{Cl}^{-}$ (increasing) occur over the initial part of the scan. The Cl• component has a shape very similar to $\mathrm{CHCl}_{3}$ except for $\alpha<0.43^{\circ}$. We interpret this as $\mathrm{Cl} \bullet$ being a damage product of $\mathrm{CHCl}_{3}$ which undergoes further beam damage to produce $\mathrm{Cl}^{-}$. This means that $\mathrm{Cl} \bullet$ is a reactive intermediate which will have a low concentration equal to a fixed fraction of the $\mathrm{CHCl}_{3}$ concentration. The reaction sequence assuming the secondary electron flux as the cause of damage is

$$
\begin{gathered}
\mathrm{CHCl}_{3}+\mathrm{e}^{-} \rightarrow \mathrm{Cl} \bullet+\text { other products } \\
\mathrm{Cl} \bullet+\mathrm{e}^{-} \rightarrow \mathrm{Cl}^{-}
\end{gathered}
$$

As the sample is at $90 \mathrm{~K}$ the damage products are unlikely to be able to diffuse more than a few $\AA$ from the position of the original $\mathrm{CHCl}_{3}$ species. The intensities of the three species have therefore been summed, Fig.12A, to produce a single VPXSW curve for fitting.

\section{VPXSW of the $\mathrm{CHCl}_{3}-\mathrm{H}_{2} \mathrm{O}$ marker layer}

Figure 12B shows the $\mathrm{Cl} 1 \mathrm{~s}_{\text {tot }}$ and $\mathrm{O} 1 \mathrm{~s}_{\text {tot }}$ VPXSW scans, normalised to 1 at their maxima, versus $\alpha$. Within experimental error both curves are the same, as expected if the $\mathrm{H}_{2} \mathrm{O}$ and $\mathrm{CHCl}_{3}$ are in a thin marker layer on top of the IL spacer layer. Both exhibit a rapid rise for $\alpha<\alpha_{2}$, crit due to the high intensity part of the standing wave reaching the outmost parts of the surface first, Fig.12B, and both signals oscillate for $\alpha>\alpha_{2, \text { crit }}$ because both layers are thin and on the outside of the IL spacer layer where variations in the X-ray standing wave intensity are most pronounced. The best fit is 
shown in Fig. 12B for a combined marker layer of $\mathrm{CHCl}_{3}$ and $\mathrm{H}_{2} \mathrm{O}$ with $t=12 \pm 10 \AA$ thick, with its outer surface located at $223 \pm 15 \AA$ from the substrate surface, see schematic in Fig. 6B. The simulation used $\lambda=9 \AA$ for $\mathrm{Cl} 1 \mathrm{~s}$ photoelectrons and $\lambda=$ $55 \AA$ for the $\mathrm{O} 1 \mathrm{~s}$ photoelectrons. A full estimation of errors is given in $\mathrm{C}$ in the supplemental material ${ }^{40}$. The experimental data is less modulated than the simulation at the second oscillation suggesting a level of disorder within the experimental surface which is not simulated. Disorder, either static or dynamic (i.e. thermal), or both, could be incorporated into the simulations, but for the present study the simulations maintain perfect ordering.
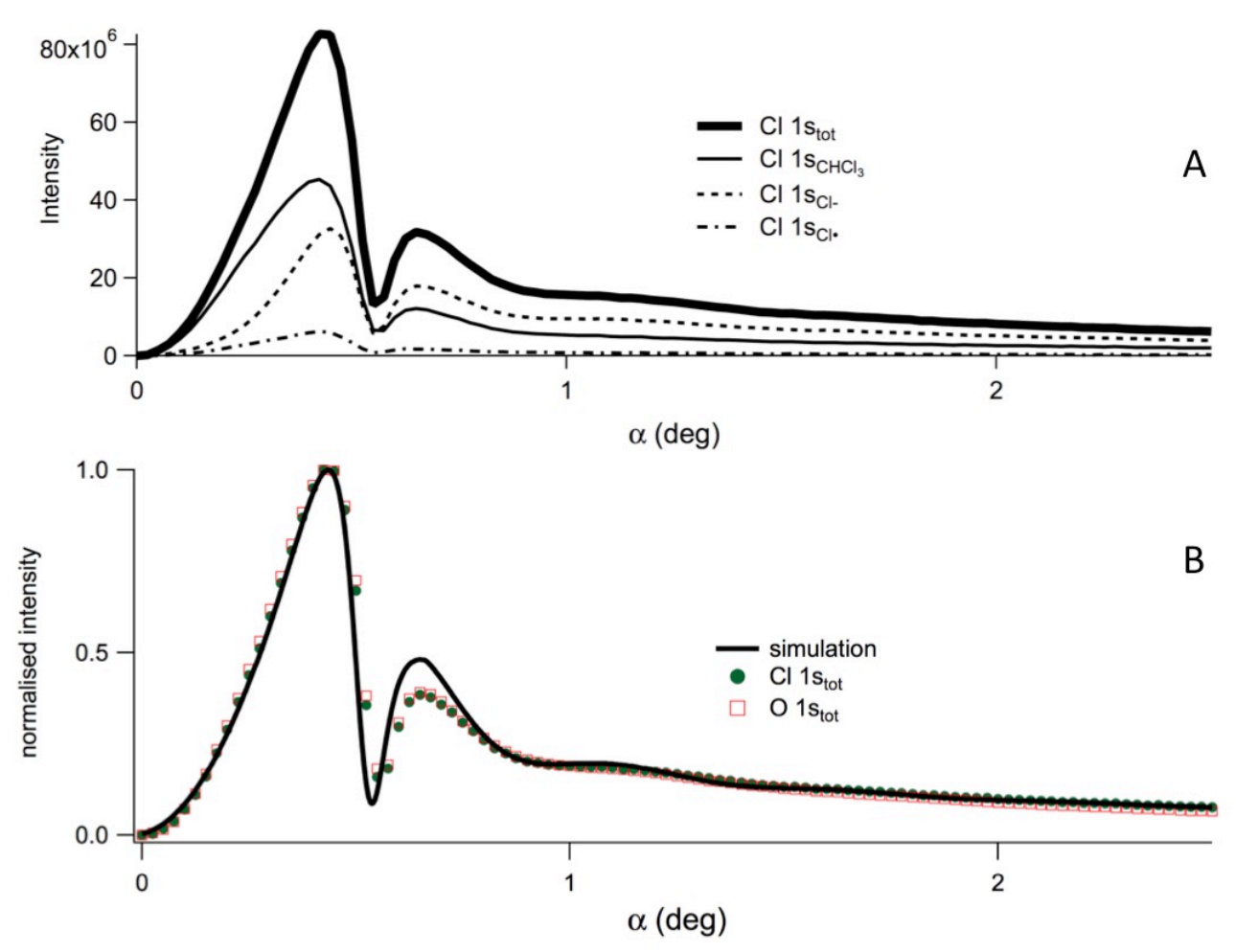

Figure 12. VPXSW scans for the multilayer surface obtained using $\mathrm{Cl}$ and $\mathrm{O} 1 \mathrm{~s}$ photoelectron peaks. A, intensity versus $\alpha$ for the $\mathrm{Cl} 1 \mathrm{~s}_{\mathrm{CHCl}}, \mathrm{Cl} 1 \mathrm{~s}_{\mathrm{Cl}}{ }^{-}, \mathrm{Cl} 1 \mathrm{~s}_{\mathrm{Cl}}$. and $\mathrm{Cl} 1 \mathrm{~s}_{\text {tot. }} \mathrm{B}, \mathrm{Cl} 1 \mathrm{~s}_{\text {tot }}$ and $\mathrm{O} 1 \mathrm{~s}_{\text {tot }}$ intensities and the best fit VPXSW simulation for a surface consisting of a $\mathrm{CHCl}_{3}-\mathrm{H}_{2} \mathrm{O}$ layer $12 \AA$ thick with its outer surface $223 \AA$ away from the $\mathrm{SiO}_{2}$ surface. Simulation (corrected for footprint, path length and shifted by $0.025^{\circ}$ ) used $\lambda=9 \AA$. 
The VPXSW curves obtained using the two components of carbon from the IL, C $1 \mathrm{~s}_{\mathrm{alkyl}}$ and $\mathrm{C} 1 \mathrm{~s}_{\text {hetero, }}$, are shown in Fig. 13A. Although similar, they differ in relative intensities below $0.5^{\circ}$ which is consistent with a build up of beam damage products within the envelope of peaks in the EDCs (see , Fig. 9A) which disturbs the apparent relative amounts of the two carbon components. Using the same assumption as for chloroform, above, that the damage products are unlikely to move more than a few $\AA$ from their site of production, the total carbon signal, $\mathrm{C} 1 \mathrm{~s}_{\mathrm{tot}}$, has been used for subsequent analysis.

The VPXSW curves for carbon $\left(\mathrm{C} 1 \mathrm{~s}_{\mathrm{tot}}\right)$, fluorine $(\mathrm{F} 1 \mathrm{~s})$, nitrogen $\left(\mathrm{N} 1 \mathrm{~s}_{\mathrm{tot}}\right)$ and boron (B 1s) are shown in Fig. 13B, where $\mathrm{N} 1 \mathrm{~s}_{\text {tot }}$ is the total intensity of the nitrogen peak, and fluorine and boron are just the intensities of the single peaks. The $\mathrm{C}, \mathrm{N}$ and $\mathrm{F}$ VPXSW scans are the same within experimental error, while the B curve is similar, but has a slower onset at low angles and less of a peak at $\approx 0.7^{\circ}$. We attribute the similarity of the $\mathrm{C}, \mathrm{N}$ and $\mathrm{F}$ scans to the IL spacer layer being homogeneous, consisting of randomly orientated ion pairs which stuck where they landed at the deposition temperature of $90 \mathrm{~K}$, giving a flat distribution of the elements across the depth of the layer. The best fit to the C, N and F curves, Fig.13B, was obtained for a total thickness of $223 \pm 15 \AA$ consisting of an IL slab of thickness $211 \AA$, with a photoelectron empty marker layer of $\mathrm{CHCl}_{3}-\mathrm{H}_{2} \mathrm{O} 12 \pm 10 \AA$ thick on top, using an average value of $\lambda=55 \AA$. Although the marker layer does contain carbon from the $\mathrm{CHCl}_{3}$, the carbon density is $<0.27$ of that in the spacer layer, and it was not detected in the EDCs, so here it has been set equal to zero. An estimation of errors can be found in $\mathrm{C}$ in the supplemental material ${ }^{40}$. These distances are entirely consistent with the VPXSW analysis from the $\mathrm{CHCl}_{3}$ and $\mathrm{H}_{2} \mathrm{O}$ marker layers.

The VPXSW scan for boron might have been expected to behave the same as for C, N, and $\mathrm{F}$, as it too would have been deposited evenly throughout the spacer layer. However, the boron curve rises more slowly than the other components in the IL at low angles and has a less pronounced peak at $0.7^{\circ}$. This would be consistent with the loss of $\mathrm{BF}_{3}$ gas from the surface ${ }^{57}$. The anion $\left[\mathrm{BF}_{4}\right]^{-}$can react with holes generated by the X-radiation ${ }^{58}$ to form $\mathrm{BF}_{3}$ which is sufficiently volatile at $90 \mathrm{~K}$ to desorb. This would have the effect of reducing the amount of boron in the top-most layers of the IL spacer layer, equivalent to increasing the depth of the marker layer for which no 
boron is present. Figure 13B shows a VPXSW simulation where the boron in the spacer layer has been reduced to a thickness of $181 \AA$ while keeping the total adlayer thickness at $223 \AA$. The fit is quite good, supporting the loss of boron from the top $\approx$ $30 \AA$ of the IL spacer layer as being the cause for the different behaviour of this curve. See Fig.6B for a cartoon of the surface, and B in the supplemental material ${ }^{40}$ for a description of the beam damage to $\left[\mathrm{BF}_{4}\right]^{-}$.
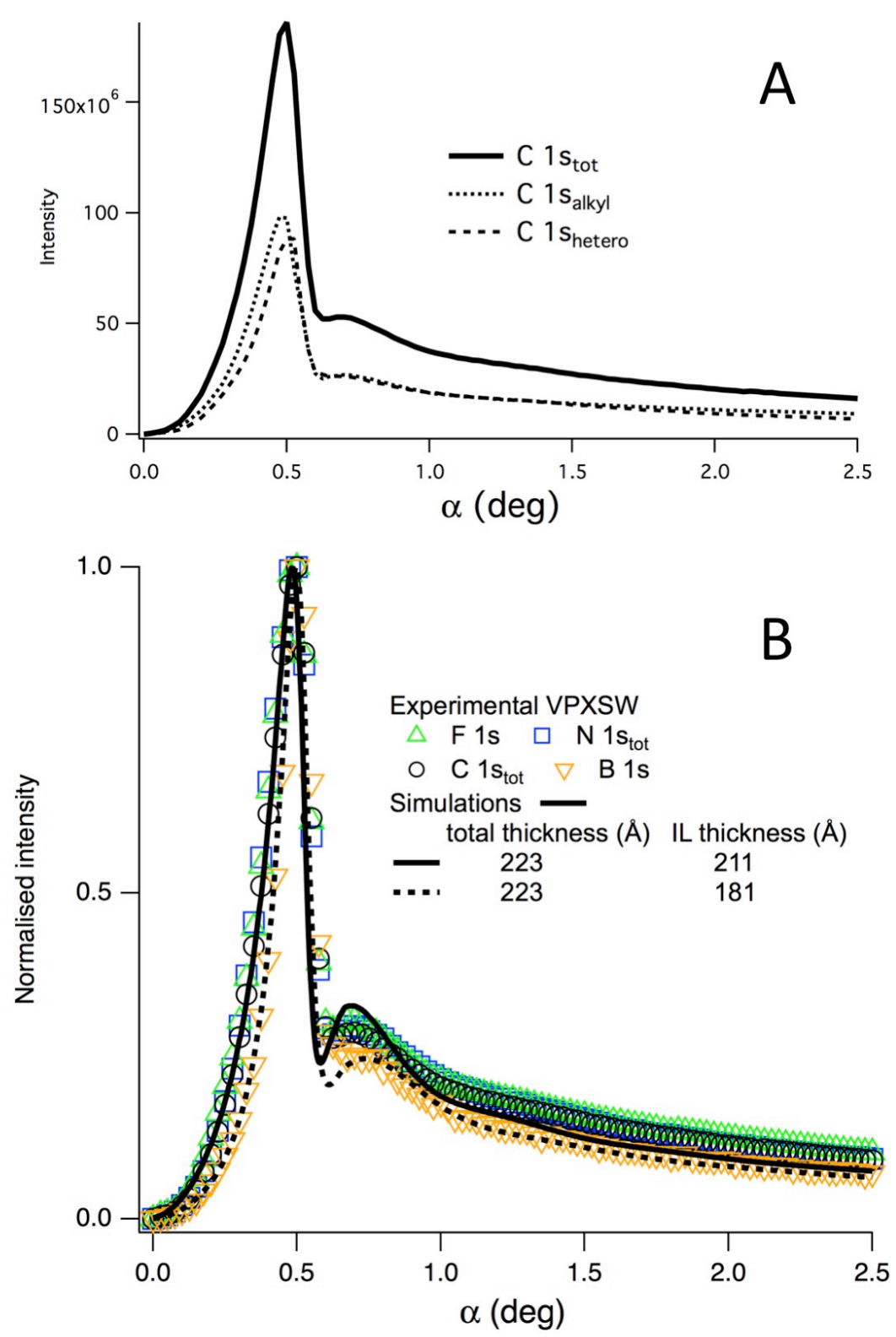

Figure 13. Experimental and simulated VPXSW curves for the IL layer. A, experimental data using the $\mathrm{C} 1 \mathrm{~s}_{\mathrm{alkyl}}$ and $\mathrm{C} 1 \mathrm{~s}_{\text {hetero }}$ components and their sum, $\mathrm{C} 1 \mathrm{~s}_{\text {tot. }}$. 
$\mathrm{B}$, experimental data using $\mathrm{F} 1 \mathrm{~s}, \mathrm{~N} 1 \mathrm{~s}_{\mathrm{tot}}, \mathrm{C} 1 \mathrm{~s}_{\mathrm{tot}}$ and $\mathrm{B} 1 \mathrm{~s}$ photoelectron intensities and simulations using a total thickness of $223 \AA$ and IL layer thicknesses of $211 \AA$ and $181 \AA$. Simulations (corrected for X-ray footprint, path length and shifted by $0.025^{\circ}$ ) used $\lambda=55 \AA$.

The silicon peaks from the $\mathrm{Si}_{-} \mathrm{SiO}_{2}$ substrate were sufficiently penetrating that they had a measurable intensity when a higher pass energy $(500 \mathrm{eV})$ was used on the CHA. VPXSW cans were taken using the Si $2 p$ photoelectron peaks, and fitted using $2 p_{3 / 2}$ and $2 p_{1 / 2}$ components for both the bulk and oxide. Figure 14 shows the VPXSW curves plotted as the sum of the spin-orbit split components for the bulk and the oxide. Both have almost no intensity below the critical angle of the organic layer, and then rise rapidly to a maximum at the silicon critical angle. To higher angles the oxide signal drops more quickly than the bulk signal due to the X-rays penetrating into the bulk silicon at greater angles and hence producing a greater flux of photoelectrons. Also shown in Fig.14 are two simulated curves. Both used a total organic layer thickness of $223 \AA$, as derived from the above analysis, a $\lambda$ value of $58 \AA$ through the bulk silicon and $\mathrm{SiO}_{2}$ and a slightly increased X-ray beam diameter to CHA focal spot diameter ratio of 0.015 due to the higher pass energy used. The attenuation of both the bulk $\mathrm{Si}$ and $\mathrm{SiO}_{2}$ photoelectron fluxes as they pass through the overlying organic layer remains constant with respect to angle, and hence need not be calculated making the value of $\lambda$ for the organic layer irrelevant here. For the oxide simulation a marker layer $20 \AA$ thick was used (as determined above), lying between the bulk silicon and the organic layer. The fits to both the oxide and the bulk curves are good. The kinks in both simulations at $\approx 0.85^{\circ}$ are due to the rather simple footprint correction used here, the value of the correction changing abruptly as the X-ray footprint exceeds the CHA field of view at low angles, causing the kink. A more realistic, smoothed, transition could be used in later work. From this it can be seen that the thicknesses found using the $\mathrm{CHCl}_{3}-\mathrm{H}_{2} \mathrm{O}$ marker layer signals and the ionic liquid spacer layer signals, also fit the signals from the bulk silicon and the $\mathrm{SiO}_{2}$ layer that lie below the organic layer. 


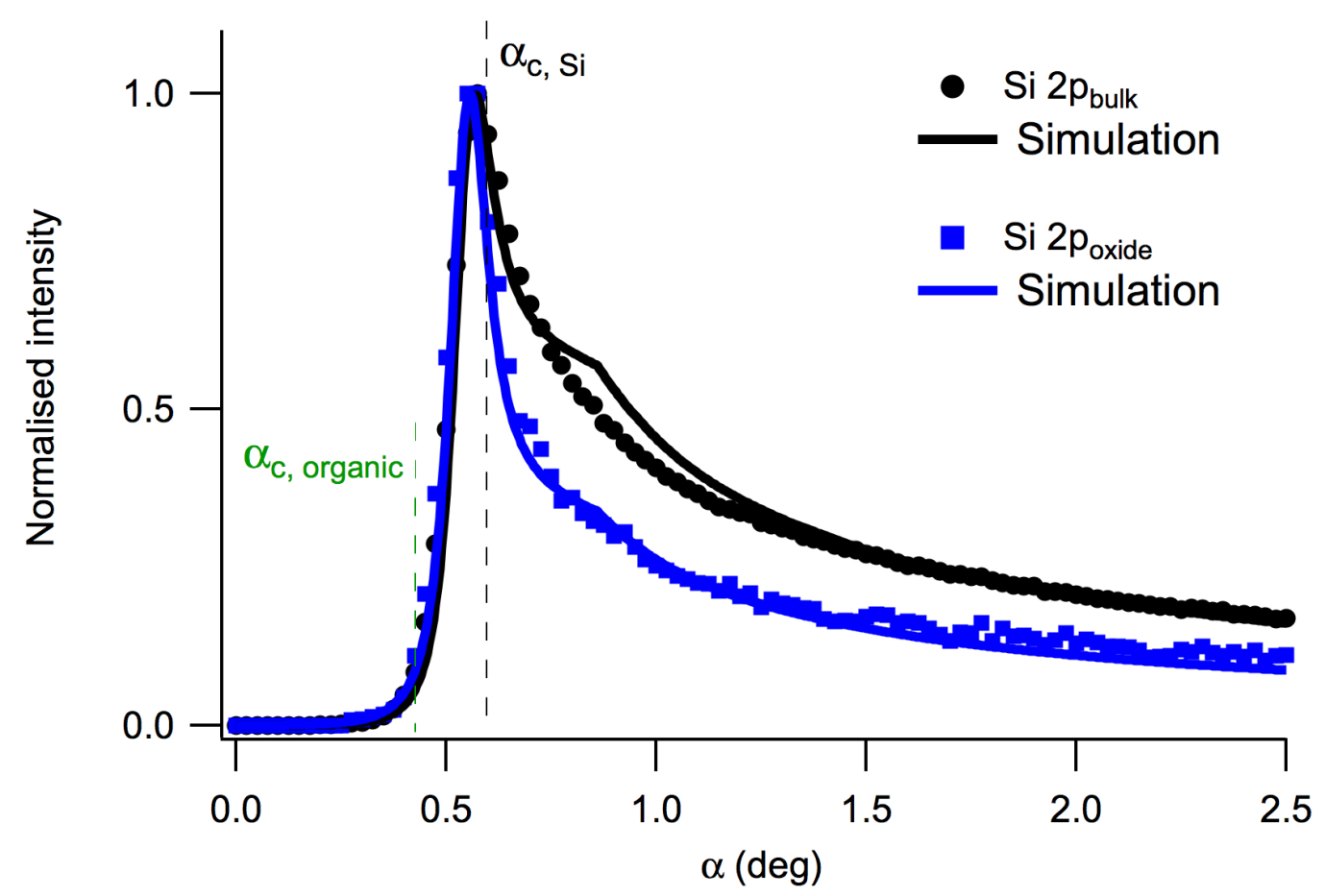

Figure 14. VPXSW scans of Si $2 p$ intensities (sum of $2 p_{3 / 2}$ and $2 p_{1 / 2}$ ) for bulk Si bulk and $\mathrm{SiO}_{2}$ lying beneath the organic layer, sample 2. The simulations (normalised to 1 , corrected for footprint and path length) used $\lambda=58 \AA$ for transmission through bulk Si and $\mathrm{SiO}_{2}$, an oxide layer $20 \AA$ thick, and an organic layer $223 \AA$ thick. See cartoon in Fig. 6B. The critical angles of the bulk silicon and organic layers are also shown.

\section{Surface Roughness}

For the photoelectron signals originating from the $\mathrm{Si}_{-} \mathrm{SiO}_{2}$ surface in contact with the vacuum (sample 1) or in contact with the spacer layer above it (sample 2), there were no discernible differences between the calculated VPXSW curve (which uses perfectly flat interfaces) and experimental VPXSW curves (where there must be interface roughness). This indicates that any effects due to interface roughness were not apparent due to the experimental noise. However, for photoelectron signals from the ionic liquid spacer layer and $\mathrm{CHCl}_{3} / \mathrm{H}_{2} \mathrm{O}$ marker layer VPXSW curves, Figs 12 and 13 , the intensity variations in the experiment were less than those in the simulations indicating an observable effect of roughness in the spacer layer/marker layer/vacuum interfaces. Roughness in reflectivity measurements is usually defined as a Gaussian distribution of surface facet heights about the mean average surface for the substrate, with a root mean square value of $\sigma$. It can be defined for each interface in the system, with (usually) much larger distributions for adsorbate interfaces above 
the substrate. The effect of these roughness distributions on the measured fluorescence yield in variable period X-ray standing waves has been extensively discussed by Zhang et al ${ }^{25}$ who show that the VPXSW oscillations are broadened and dampened. In the present work roughness at the substrate as well as the adsorbate interfaces will affect the spatial extent of the X-ray standing wave which initiates photoelectron emission as well as affecting the measured photoelectron intensity via attenuation across the rough regions. To obtain an approximate value for the spacer layer roughness the average of nine discrete simulations for varying thicknesses of the spacer layer were calculated while keeping the marker layer thickness fixed at $12 \AA$. The average simulations approximately matched the reduced oscillations of the experimental $\mathrm{C}$ and $\mathrm{O}$ signals in the marker layer and the $\mathrm{B}, \mathrm{C}, \mathrm{N}$ and $\mathrm{F}$ signals in the spacer layer. The roughness needed to achieve this was $\sigma \approx 40 \AA$. A later work will address the roughness more accurately.

\section{CONCLUSIONS}

Variable period X-ray standing wave experiments have been carried out using photoelectron detection. $3 \mathrm{keV}$ X-rays were reflected from a polished silicon surface over an angular range of $2.5^{\circ}$. Two surfaces layer structures were studied: the native oxide of silicon on a bulk silicon substrate, and a purpose built model surface consisting of a the native oxide/silicon as a substrate, surmounted by a spacer layer of solid [OMIM] $\left[\mathrm{BF}_{4}\right]$, which terminated in a marker layer composed of $\mathrm{CHCl}_{3}$ and $\mathrm{H}_{2} \mathrm{O}$. VPXSW data was obtained by monitoring the photoelectron intensities of $\mathrm{Si} 2 \mathrm{p}_{1 / 2,3 / 2}$ and $\mathrm{O} 1 \mathrm{~s}$ for the $\mathrm{SiO}_{2} / \mathrm{Si}$ surface, and of $\mathrm{Si} 2 \mathrm{p}_{1 / 2,3 / 2}$ and $\mathrm{B}, \mathrm{C}, \mathrm{N}, \mathrm{O}, \mathrm{F}$ and $\mathrm{Cl} 1 \mathrm{~s}$ for the $\mathrm{Si}_{-} \mathrm{SiO}_{2} /[\mathrm{OMIM}]\left[\mathrm{BF}_{4}\right]-\mathrm{CHCl}_{3}-\mathrm{H}_{2} \mathrm{O}$ surface (spanning kinetic energies of 100-2830 eV). The VPXSW scans were analysed by modelling them using dynamical X-ray theory and a three layer system, vacuum/adsorbate layer/substrate layer to determine the X-ray intensities within the adsorbate and the substrate, the Beer-Lambert law and appropriate attenuation lengths to model photoelectron emission along the surface normal, and two corrections, for X-ray path length through the surface layers and the footprint of the X-ray relative to the field of view of the electron energy analyser. The $\mathrm{SiO}_{2}$ film was found to comprise a layer $20 \pm 10 \AA$ thick using three independent photoelectron measurements, $\mathrm{Si} 2 \mathrm{p}_{1 / 2,3 / 2}$ from the bulk and from the surface $\mathrm{SiO}_{2}$, and $\mathrm{O} 1 \mathrm{~s}$ from the $\mathrm{SiO}_{2}$. For the combined $\mathrm{CHCl}_{3} / \mathrm{H}_{2} \mathrm{O}$ marker layer surface, the 
thickness $(12 \pm 10 \AA)$ and position of the outside surface $(223 \pm 15 \AA)$ were found using the high energy $\mathrm{O} 1 \mathrm{~s}$ and the low energy $\mathrm{Cl} 1 \mathrm{~s}$ photoelectron peaks. The thickness of the ionic liquid spacer layer was found to be $211 \pm 15 \AA$ using the C, N and $\mathrm{F}$ 1s photoelectron peaks. This is entirely compatible with the marker layer thickness. The total thickness of the spacer and marker layers (223 $\AA)$ determined using the bulk Si $2 p_{1 / 2,3 / 2}$ photoelectron signal that had passed through them, was consistent with the other measurements. The high intensity X-ray beam running parallel to the surface at the critical angle of the organic layer resulted in beam damage to all three components, $[\mathrm{OMIM}]\left[\mathrm{BF}_{4}\right], \mathrm{CHCl}_{3}$ and $\mathrm{H}_{2} \mathrm{O}$. The damage product, $\mathrm{BF}_{3}$, from $\left[\mathrm{BF}_{4}\right]^{-}$was thought to escape from the surface giving a boron VPXSW curve characteristic of a $30 \AA$ thick layer, depleted of boron, in the outmost part of the IL. The high flux of photoelectrons also led to substantial charging (9.3 $\mathrm{eV}$ ) of the insulating organic layer. The depth accessible to measurement here, using photoelectrons from $3 \mathrm{keV}$ photons, is $\lesssim 300 \AA$. The accuracy for determining the position of a marker layer and the thickness of a slab are about $10 \AA$ and $15 \AA$ respectively, while the accuracy of determining marker layer thicknesses is about 10 $\AA$. Chemical shifts in the photoelectron peaks clearly showed that chemical state specificity is easily achieved. Future work should be capable of determining an arbitrary density distribution through an adlayer by combining VPXSW data with different electron attenuation lengths obtained using different X-ray energies. Other possibilities also exist to carry out spatially resolved NEXAFS studies.

\section{ACKNOWLEDGEMENTS}

Funding from the Engineering and Physical Sciences ${ }^{59}$ Research Council, UK, (EP/I018093/1), synchrotron beamtime from Diamond Light Source (SI 9118) and $\mathrm{PhD}$ studentship from School of Chemistry, Nottingham (JSG) are gratefully acknowledged. We thank P. Licence for providing the ionic liquid. 


\section{References}

1 E. Langereis, S. B. S. Heil, H. C. M. Knoops, W. Keuning, M. C. M. van de Sanden, and W. M. M. Kessels, J. Phys. D: Appl. Phys. 42, 73001 (2009).

2 D. E. Aspnes, Thin Solid Films 571, 334 (2014).

$3 \quad$ M. Losurdo, et al., J. Nanopart. Res. 11, 1521 (2009).

4 E. Chason and T. M. Mayer, Critical Reviews in Solid State and Materials Sciences 22, 1 (1997).

$5 \quad$ J. C. Vickerman and D. Briggs, ToF-SIMS: Materials Analysis by Mass Spectrometry (IM Publications Chichester, UK and Surface Spectra Limited Manchester, UK, 2013).

P. J. Cumpson, J. Elecron. Spec. and Rel. Phenom. 73, 25 (1995).

$7 \quad$ J.Zegenhagen and A. Kazimirov eds., The X-ray standing wave technique Principles and Applications (World Scientific, Singapore, 2013).

D. P. Woodruff, Reports on Progress in Physics 68, 743 (2005).

S. H. Yang, A. X. Gray, A. M. Kaiser, B. S. Mun, B. C. Sell, J. B. Kortright, and C. S. Fadley, Journal of Applied Physics 113, 25 (2013).

C. Papp, G. Conti, B. Balke, S. Ueda, Y. Yamashita, H. Yoshikawa, Y. S. Uritsky, K. Kobayashi, and C. S. Fadley, Journal of Applied Physics 112, 8 (2012).

A. von Bohlen, Spectrochimica Acta Part B-Atomic Spectroscopy 64, 821 (2009).

R. G. Jones, et al., Journal of Physics-Condensed Matter 14, 4059 (2002).

R. G. Jones, et al., in Physics of Low-Dimensional Structures (VSV Co.Ltd, Moscow, 2001), p. 1.

D. P. Woodruff, Prog. Surf. Sci. 57, 1 (1998).

J. Zegenhagen, Surface Science Reports 18, 202 (1993).

D. P. Woodruff, B. C. C. Cowie, and A. Ettema, Journal of PhysicsCondensed Matter 6, 10633 (1994).

M. J. Bedzyk, G. M. Bommarito, and J. S. Schildkraut, Phys. Rev. Lett. 62, 1376 (1989).

M. J. BEDZYK, in The X-Ray Standing Wave Technique (World Scientific, Singapore, 2013), p. 94.

Z. Erdelyi, et al., X-Ray Spectrom. 38, 338 (2008).

R. Itri, R. T. Zhang, and M. Caffrey, Biophys. J. 73, 1506 (1997).

M. Kraemer, A. von Bohlen, C. Sternemann, M. Paulus, and R.

Hergenroeder, Journal of Analytical Atomic Spectrometry 21, 1136 (2006).

M. Kraemer, A. von Bohlen, C. Sternemann, M. Paulus, and R. Hergenroeder, Appl. Surf. Sci. 253, 3533 (2007). J. Wang, M. J. Bedzyk, T. L. Penner, and M. Caffrey, Nature 354, 377 (1991). J. Wang, M. Caffrey, M. J. Bedzyk, and T. L. Penner, J. Phys. Chem. 98, 10957 (1994).

R. T. Zhang, R. Itri, and M. Caffrey, Biophys. J. 74, 1924 (1998).

M. Mehta and C. S. Fadley, Physics Letters A 55, 59 (1975).

M. Mehta and C. S. Fadley, Chemical Physics Letters 46, 225 (1977). 
J. Kawai, M. Takami, M. Fujinami, Y. Hashiguchi, S. Hayakawa, and Y. Gohshi, Spectrochimica Acta Part B-Atomic Spectroscopy 47, 983 (1992). J. Kawai, S. Hayakawa, Y. Kitajima, K. Maeda, and Y. Gohshi, Journal of Electron Spectroscopy and Related Phenomena 76, 313 (1995). J. Kawai, S. Kawato, K. Hayashi, T. Horiuchi, K. Matsushige, and Y. Kitajima, Applied Physics Letters 67, 3889 (1995). J. Kawai, Journal of Electron Spectroscopy and Related Phenomena 178179, 268 (2010).

M. J. Chester and T. Jach, Physical Review B 48, 17262 (1993).

M. J. Chester, T. Jach, and S. Thurgate, J. Vac. Sci. Technol. B 11, 1609 (1993).

T. Jach, J. Gormley, and S. Thurgate, Spectrochimica Acta Part B-Atomic Spectroscopy 54, 1539 (1999).

T. Jach and E. Landree, Journal of Surface Analysis 9, 339 (2002).

T. Jach, J. A. Dura, N. V. Nguyen, J. Swider, G. Cappello, and C. Richter, Surf. Interface Anal. 36, 23 (2004).

E. Landree, T. Jach, D. Brady, A. Karamcheti, J. Canterbury, W. Chism, and A. C. Diebold, in Characterization and Metrology for Ulsi Technology 2000, International Conference, edited by D. G. Seller, A. C. Diebold, T. J. Shaffner, R. McDonald, W. M. Bullis, P. J. Smith and E. M. Secula (Amer Inst Physics, Melville, 2001), Vol. 550, p. 159.

D. P. Woodruff and T. A. Delchar., Modern Techniques of Surface Science (Cambridge University Press, Cambridge, UK, 1994).

B. L. Henke, E. M. Gullikson, and J. C. Davis, Atom. Data Nucl. Data Tables 54, 181 (1993).

See supplemental material at http://link.aps.org/supplemental/10.1103/PhysRevB.00.005400 for A, X-ray constants, B, possible desorption of BF3 damage products from a $90 \mathrm{~K}$ surface and C, estimate of errors in fittings.

D. Briggs and J. T. Grant eds., Surface Analysis by Auger and X-ray Photoelectron Spectroscopy. (IM Publications, Chichester, 2003). C. J. Powell and A. Jablonski, NIST Electron Inelastic-Mean-Free-Path Database - Version 1.2 (National Institute of Standards and Technology, Gaithersburg, MD 2010). R. G. Jones, (2016). R. Foulston, S. Gangopadhyay, C. Chiutu, P. Moriarty, and R. G. Jones, Phy. Chem. Chem. Phys. 14, 6054 (2012).

M. Buckley, Nottingham, PhD 2016.

C. J. Fisher, R. Ithin, R. G. Jones, G. J. Jackson, D. P. Woodruff, and B. C. C. Cowie, Journal of Physics-Condensed Matter 10, L623 (1998).

J. J. Lee, C. J. Fisher, D. P. Woodruff, M. G. Roper, R. G. Jones, and B. C. C. Cowie, Surface Science 494, 166 (2001).

R. G. Jones, (2016).

D. N. Belton, S. J. Harris, S. J. Schmieg, A. M. Weiner, and T. A. Perry, Applied Physics Letters 54, 416 (1989).

G. B. Hoflund, D. A. Asbury, C. F. Corallo, and G. R. Corallo, Journal of Vacuum Science \& Technology a-Vacuum Surfaces and Films 6, 70 (1988). C. J. Powell and A. Jablonski, Journal of Physical and Chemical Reference Data 28, 19 (1999). 
A. C. Thompson and D. Vaughan eds., X-ray data booklet (Lawrence Berkeley National Laboratory, Berkeley, California, USA, 2001). E. F. Smith, F. J. M. Rutten, I. J. Villar-Garcia, D. Briggs, and P. Licence, Langmuir 22, 9386 (2006). I. J. Villar-Garcia, E. F. Smith, A. W. Taylor, F. Qiu, K. R. J. Lovelock, R. G. Jones, and P. Licence, Physical Chemistry Chemical Physics 13, 2797 (2011).

U. Gelius, P. F. Hedén, J. Hedman, B. J. Lindberg, R. Manne, R. Nordberg, C. Nordling, and K. Siegbahn, Physica Scripta 2, 70 (1970). K. R. J. Lovelock, E. F. Smith, A. Deyko, I. J. Villar-Garcia, P. Licence, and R. G. Jones, Chem. Commun., 4866 (2007).

NIST, in https://webbook.nist.gov/chemistry/, p. Standard Reference Database Number 69

I. A. Shkrob, T. W. Marin, S. D. Chemerisov, and J. F. Wishart, J. Phys. Chem. B 115, 3872 (2011).

R. A. Fass and S. G. Kendall, Journal of Chemical Education 48, 545 (1971). 
